\title{
UK Renal Registry 15th Annual Report: Chapter 3 Demographic and Biochemistry Profile of Kidney Transplant Recipients in the UK in 2011: National and Centre-Specific Analyses
}

\author{
Rishi Pruthi ${ }^{a}$, Anna Casula $^{a}$, lain MacPhee ${ }^{b}$

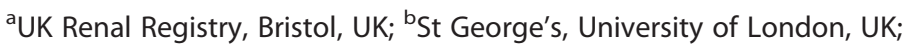

\section{Key Words}

Blood pressure - Bone metabolism - Chronic kidney disease . Deceased donor · eGFR · Epidemiology · Ethnicity · Graft function - Haemoglobin - Live donor - Outcomes - Renal transplantation · Survival

\footnotetext{
Abstract

Introduction: National transplant registries routinely focus on centre-specific patient and graft survival rates following renal transplantation. However other outcomes such as graft function (as measured by eGFR), haemoglobin and blood pressure are also important quality of care indicators. Methods: Renal transplant activity, incident graft survival data and donor information were obtained from NHS Blood and Transplant. Laboratory and clinical variables and prevalent survival data were obtained from the UK Renal Registry. Data were analysed separately for prevalent and one year post-transplant patients. Results: The only increase in transplant activity in 2011 was the use of
}

donors after circulatory death. The death-censored graft failure rate was similar to previous years at $2.2 \%$ and the transplant patient death rates remained stable at 2.3 per 100 patient years. There was centre variation in outcomes including eGFR and haemoglobin in prevalent and 1 year post-transplant patients. Analysis of prevalent transplants by chronic kidney disease stage showed $13.6 \%$ with an eGFR $<30 \mathrm{ml} / \mathrm{min} / 1.73 \mathrm{~m}^{2}$ and $1.7 \%$ with an eGFR $<15 \mathrm{ml} / \mathrm{min} / 1.73 \mathrm{~m}^{2}$. Of those with CKD stage $5 \mathrm{~T}, 34 \%$ had haemoglobin concentrations $<10.0 \mathrm{~g} / \mathrm{dl}, 19.8 \%$ phosphate concentrations $\geqslant 1.8 \mathrm{mmol} / \mathrm{L}$ and $7.1 \%$ adjusted calcium concentrations $\geqslant 2.6 \mathrm{mmol} / \mathrm{L}$. Infection (23\%), malignancy $(21 \%)$, and cardiac disease $(16 \%)$ remained amongst the commonest causes of death in patients with a functioning renal transplant. Conclusion: Significant variations in clinical outcomes (unadjusted for patientspecific variables) amongst kidney transplant recipients continued to exist in the UK and may reflect differences in healthcare delivery between renal centres.

$\begin{array}{ll}\text { KARGER } & \text { C } 2013 \mathrm{~S} . \text { Karger AG, Basel } \\ \text { Fax +4161306 1234 } & 1160-2110 / 13 / 1235-0055 \$ 38.00 / 0 \\ \begin{array}{l}\text { E-Mail karger@karger.ch } \\ \text { www.karger.com }\end{array} & \begin{array}{l}\text { Accessible online at: } \\ \text { www.karger.com/nec }\end{array}\end{array}$

Iain MacPhee

UK Renal Registry, Southmead Hospital, Southmead Road, Bristol, BS10 5NB, UK

Email: renalregistry@renalregistry.nhs.uk 


\section{Introduction}

This chapter includes independent analyses regarding renal transplant activity and survival data from the UK Transplant Registry, held by the Organ Donation and Transplantation Directorate (ODT) of NHS Blood and Transplant (NHSBT). The UK Renal Registry (UKRR) has performed additional analyses of renal transplant recipient follow-up data examining demographics, clinical and biochemical variables. NHSBT records all the information regarding the episode of transplantation (donor and recipient details) and the UKRR holds additional information on key clinical and biochemical variables in renal transplant recipients. The co-operation between these two organisations results in a comprehensive database describing the clinical care delivered to renal transplant patients within the UK. This further allows for the comparison of key outcomes between centres and provides insight into the processes involved in the care of such patients in the UK.

This chapter is divided into six sections: (1) transplant activity, waiting list and survival data; (2) transplant demographics; (3) clinical and laboratory outcomes; (4) analysis of prevalent patients by chronic kidney disease (CKD) stage; (5) eGFR slope analysis; and (6) causes of death in transplant recipients. Methodology, results and conclusions of these analyses are discussed in detail for all six sections separately.

The UK Renal Registry methodology is described elsewhere [1]. The UKRR collects quarterly clinical data via an electronic data extraction process from hospital based renal IT systems on all patients receiving renal replacement therapy. Throughout the chapter, the number preceding the centre name in each figure indicates the percentage of missing data for that centre for that variable.

Unless otherwise specified, prevalent transplant patients were defined as patients with a functioning renal transplant on the 31st December 2011.

\section{Transplant activity, waiting list activity and survival data}

\section{Introduction}

NHSBT prospectively collects donor and recipient data around the episode of transplantation. They also request transplant centres provide an annual paper based data return on the status of the recipient's graft function. This enables ODT to generate comprehensive analyses of renal transplant activity and graft survival statistics.

NHSBT attributes a patient to the centre that performed the transplant operation irrespective of where the patient was cared for before or after the procedure and hence only reports on transplant centre performance.

\section{Methods}

In 2011, there were 23 UK adult renal transplant centres, 19 in England, 2 in Scotland and 1 each in Northern Ireland and Wales.

Comprehensive information from 1999 onwards concerning the number of patients on the transplant waiting list, the number of transplants performed, the number of deceased kidney donors (donor after brainstem death and donor after circulatory death), living kidney donors, patient survival and graft survival is available on the NHSBT website (http://www. organdonation.nhs.uk/ukt/statistics/statistics.asp).

\section{Results}

During 2011, 2,752 kidney or kidney plus other organ transplants were performed. The absolute number of living kidney donors showed little change in 2011 representing $37.3 \%$ of all transplants performed whilst donor after circulatory death transplants continued to increase and comprised $21.6 \%$ of all kidney transplants performed. The rise in numbers of transplants from donors after brainstem death noted in 2010 was reversed in 2011, showing a 4\% decline (table 3.1).

There were small differences in one and five year riskadjusted patient and graft survival rates amongst UK renal transplant centres (table 3.2). These graft survival rates include grafts with primary non-function (which are excluded from analysis by some countries).

Table 3.1. Kidney and kidney plus other organ transplant numbers in the UK, 1/1/2009-31/12/2011

\begin{tabular}{lrrrc}
\hline & & & & \% change \\
Organ & 2009 & 2010 & 2011 & $2010-2011$ \\
\hline Donor after brainstem death $^{\mathrm{a}}$ & 944 & 989 & 951 & -4 \\
Donor after circulatory death $^{\mathrm{b}}$ & 496 & 549 & 594 & 8 \\
Living donor kidney $^{\text {Kidney and liver }}$ & 983 & 1,027 & 1,026 & 0 \\
Kidney and heart $_{\text {Kidney and pancreas }}^{\mathrm{c}}$ & 15 & 9 & 16 & 78 \\
Small bowel (inc kidney) $_{\text {Total kidney transplants }}^{1}$ & 0 & 0 & \\
& 158 & 150 & 163 & 9 \\
\hline
\end{tabular}

${ }^{a}$ Includes en bloc kidney transplants (3 in 2009, 7 in 2010, 7 in 2011) and double kidney transplants (6 in 2009, 6 in 2010, 5 in 2011)

${ }^{\mathrm{b}}$ Includes en bloc kidney transplants ( 1 in 2009, 2 in 2010, 2 in 2011) and double kidney transplants (4 in 2009, 16 in 2010, 32 in 2011)

${ }^{\mathrm{c}}$ Includes donor after circulatory death transplants (19 in 2009, 29 in 2010, 28 in 2011) 
Table 3.2. Risk-adjusted first adult kidney transplant only, graft and patient survival percentage rates for UK centres ${ }^{\star}$

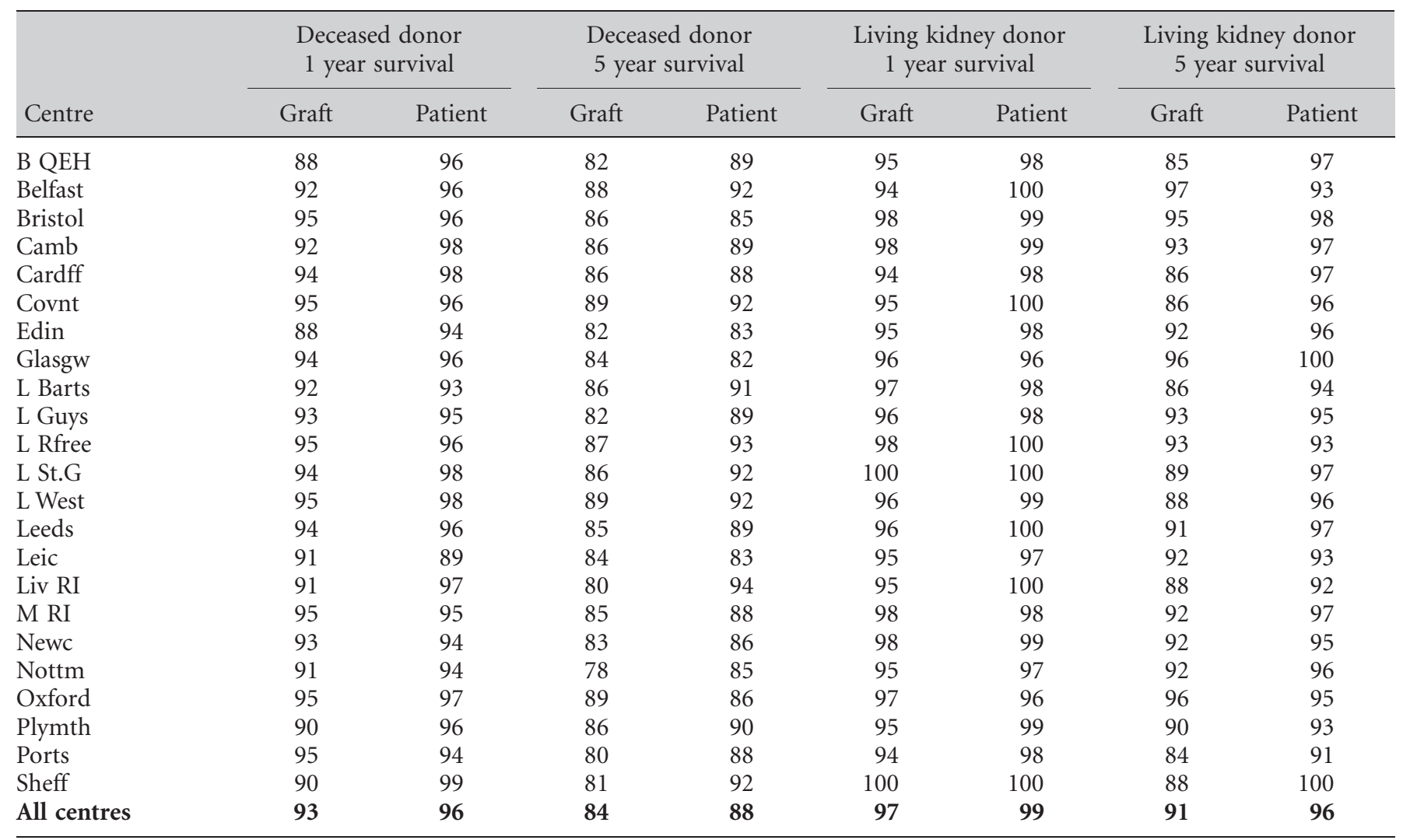

${ }^{*}$ Information courtesy of NHSBT: number of transplants, patients and 95\%CI for each estimate; statistical methodology for computing risk-adjusted estimates can be obtained from the NHSBT website (see http://www.organdonation.nhs.uk/ukt/statistics/statistics.asp)

Cohorts for survival rate estimation: 1 year survival: 1/1/2006-31/12/2010; 5 year survival: 1/1/2002-31/12/2006; first grafts only - re-grafts excluded for patient survival estimation. Since the cohorts to estimate 1- and 5-year survival are different, some centres may appear to have 5 year survival better than 1 year survival

Using data from the UKRR on prevalent renal-only transplant patients on 1st January 2011, the death rate during 2011 was 2.2/100 patient years (CI 2.0-2.4) when censored for return to dialysis and 2.3/100 patient years (CI 2.2-2.5) without censoring for dialysis. These death rates are similar to those observed over the last few years.

During 2011, 2.2\% of prevalent transplant patients experienced graft failure (excluding death as a cause of graft failure). This is the second consecutive year when graft failure rates have fallen. Whilst it might be premature to assume that graft failure rates are falling in the UK the $0.5 \%$ fall noted in the last 5 years is certainly encouraging.

\section{Conclusions}

In 2011, the increased number of kidney transplants performed was mostly due to the growing use of organs from donors after circulatory death. Graft failure rates have fallen for the second consecutive year to $2.2 \%$ per annum whilst the patient death rate of 2.3 per 100 patient years was similar to recent years.

\section{Transplant demographics}

\section{Introduction}

Since 2008, all UK renal centres have established electronic linkage to the UKRR or Scottish Renal Registry, giving the UKRR complete coverage of individual patient level data across the UK. Hope Hospital has been renamed Salford Royal and so is now abbreviated in the report as 'Salford' rather than as 'M Hope' and 'Tyrone' and 'Derry' are now grouped together as 'West NI'.

The following sections need to be interpreted in the context of variable repatriation policies; some transplant centres continue to follow up and report on all patients they transplant, whereas others refer patients back to 
non-transplant centres for most or all ongoing posttransplant care. Some transplant centres only refer back patients when their graft is failing. The time posttransplantation that a patient is referred back to their local centre varies between transplant centres. The UKRR is able to detect duplicate patients (being reported from both transplant and referring centres) and in such situations care is attributed to the referring centre. This process may result in some discrepancies in transplant numbers particularly in Oxford/Reading and Clywd/ Liverpool RI.

\section{Methods}

Three centres (Bangor, Colchester and Liverpool Aintree) did not have any transplant patients and were excluded from some of the analyses. Their dialysis patients were included in the relevant dialysis population denominators. Wirral which previously was also excluded having not had any registered transplant patients has been included in this year's report having taken on transplant patients in 2011. The nine Scottish centres only submit limited laboratory data to the UKRR and were not included in the analyses on post-transplant outcomes.

For the analysis of primary renal diagnosis (PRD) in transplant recipients, a few centres were excluded from some of the take-on years because of concerns relating to the reliability of PRD coding (with these centres submitting a high percentage of uncertain or missing aetiology codes). This year, individuals with a primary renal diagnosis (PRD) 'glomerulonephritis biopsy unproven' were grouped within the 'glomerulonephritis' PRD group, rather than within 'uncertain' (as has been the case in previous reports) to reflect better coding and bringing the registry in line with coding methodology adopted in other renal registries.

Information on patient demographics (age, gender, ethnicity and PRD) for patients in a given renal centre was obtained from UKRR patient registration data fields. Individual patients were assigned to the centre that returned data for them during 2011. The prevalence of transplant patients in areas covered by individual primary care trusts (PCT) or Health Boards/Social Care Areas (HB) was estimated based on the post code of the registered address for patients on renal replacement therapy (RRT). Data on ethnic origin, supplied as Patient Administration System (PAS) codes, were retrieved from fields within renal centre IT systems. For the purpose of this analysis, patients were grouped into Whites, South Asians, Blacks, Others and Unknown. The details of ethnicity regrouping into the above categories are provided in appendix H: Coding http://www.renalreg.com. The UKRR requires a standard set of data items regarding comorbid conditions at the time of commencement of renal replacement therapy and first registration of the patient with the UKRR.

\section{Results and discussion}

Prevalent transplant numbers across the UK are described in table 3.3.

The prevalence of renal transplant recipients in each PCT/HB in England, Northern Ireland (Health and Social Care Trust Areas), Scotland (Health Boards) and Wales (Local Health Boards) and the proportion of prevalent patients according to modality in the renal centres across the UK is described in tables 3.4 and 3.5 respectively. After standardisation for age and gender, unexplained variability was evident in the prevalence of renal transplant recipients, with some areas having higher than the predicted number of prevalent transplant patients per million population and others lower. There are a number of potential explanations for these inconsistencies, including geographical differences in access to renal transplantation in the UK which is examined in greater detail in chapter 9 Access to Transplantation.

The proportion of prevalent RRT patients with a transplant relative to the number on dialysis has been relatively stable over the last decade.

\section{Age and gender}

The gender ratio amongst incident and prevalent transplant patients has remained stable for at least the last ten years (table 3.6, figure 3.1). Note absolute patient numbers differ from those published in previous reports as a result of additional data validation and reallocation of patients. The average age of incident transplant patients has steadily increased during the same time period. There has also been a gradual increase in the average age of prevalent transplant patients, which could reflect the increasing age at which patients are transplanted and/or improved survival after renal transplantation over the last few years. The prevalent transplant patient workload across the UK had increased to 26,297 patients at the end of 2011. The continued expansion of this patient group means there is a need for careful planning by renal centres for future service provision and resource allocation.

Table 3.3. The prevalence per million population ( $\mathrm{pmp}$ ) of renal transplants in adults in the UK on $31 / 12 / 2011$

\begin{tabular}{|c|c|c|c|c|c|}
\hline & England & $\mathrm{N}$ Ireland & Scotland & Wales & UK \\
\hline All UK centres & 22,011 & 707 & 2,197 & 1,382 & 26,297 \\
\hline Total population, mid-2011 estimates from $\mathrm{ONS}^{\star}$ (millions) & 53.0 & 1.8 & 5.3 & 3.1 & 63.2 \\
\hline Prevalence pmp transplant & 415 & 390 & 415 & 451 & 416 \\
\hline
\end{tabular}

\footnotetext{
* Office of National Statistics, UK
} 
Table 3.4. The prevalence per million population ( $\mathrm{pmp}$ ) of patients with a renal transplant and standardised rate ratio in the UK, as on 31st December 2007-2011

${ }^{a}$ PCT/HB = Primary Care Trust (England); Health and Social Care Trust Areas (Northern Ireland); Health Board (Scotland) and Local Health Board (Wales)

${ }^{\mathrm{b}}$ Population numbers based on the 2010 mid-year estimates by age group and gender (data obtained from the Office of National Statistics)

${ }^{\mathrm{c}} \mathrm{O} / \mathrm{E}=$ age and gender standardised acceptance rate ratio

PCTs with significantly high average rate ratios are bold in greyed areas

PCTs with significantly low average rate ratios are italicised in greyed areas

Blank cells $=$ no data returned to the UKRR for that year

LCL $=$ lower $95 \%$ confidence limit

$\mathrm{UCL}=$ upper $95 \%$ confidence limit

\begin{tabular}{|c|c|c|c|c|c|c|c|c|c|c|}
\hline \multirow[b]{2}{*}{ UK Area } & \multirow[b]{2}{*}{$\mathrm{PCT} / \mathrm{HB}^{\mathrm{a}}$} & \multirow{2}{*}{$\begin{array}{l}\text { Population } \\
\text { covered }^{\text {b }}\end{array}$} & \multicolumn{5}{|c|}{ Rate pmp } & \multicolumn{3}{|c|}{$\begin{array}{c}\text { Age and gender } \\
\text { standardised rate ratio } 2011\end{array}$} \\
\hline & & & 2007 & 2008 & 2009 & 2010 & 2011 & $\mathrm{O} / \mathrm{E}^{\mathrm{c}}$ & LCL & UCL \\
\hline \multirow[t]{12}{*}{ North East } & County Durham & 510,800 & 378 & 390 & 397 & 413 & 431 & 0.99 & 0.86 & 1.12 \\
\hline & Darlington & 100,600 & 358 & 378 & 338 & 368 & 417 & 0.97 & 0.72 & 1.31 \\
\hline & Gateshead & 192,000 & 380 & 391 & 406 & 411 & 432 & 1.00 & 0.81 & 1.24 \\
\hline & Hartlepool & 91,400 & 394 & 361 & 350 & 394 & 405 & 0.96 & 0.69 & 1.32 \\
\hline & Middlesbrough & 142,100 & 380 & 415 & 450 & 457 & 514 & 1.29 & 1.03 & 1.62 \\
\hline & Newcastle & 292,200 & 359 & 359 & 366 & 366 & 387 & 1.02 & 0.84 & 1.22 \\
\hline & North Tyneside & 198,400 & 484 & 494 & 514 & 565 & 590 & 1.35 & 1.13 & 1.62 \\
\hline & Northumberland & 312,100 & 401 & 407 & 407 & 391 & 442 & 0.96 & 0.81 & 1.13 \\
\hline & Redcar and Cleveland & 137,300 & 495 & 524 & 539 & 546 & 554 & 1.26 & 1.01 & 1.58 \\
\hline & South Tyneside & 154,100 & 422 & 422 & 428 & 415 & 461 & 1.07 & 0.85 & 1.35 \\
\hline & Stockton-on-Tees Teaching & 192,600 & 337 & 384 & 400 & 395 & 384 & 0.90 & 0.72 & 1.14 \\
\hline & Sunderland Teaching & 283,400 & 399 & 409 & 399 & 413 & 455 & 1.06 & 0.89 & 1.26 \\
\hline \multirow[t]{24}{*}{ North West } & Ashton, Leigh and Wigan & 307,200 & 348 & 361 & 342 & 394 & 462 & 1.06 & 0.90 & 1.25 \\
\hline & Blackburn with Darwen Teaching & 140,000 & 314 & 321 & 329 & 329 & 371 & 0.96 & 0.73 & 1.26 \\
\hline & Blackpool & 140,200 & 285 & 335 & 342 & 342 & 342 & 0.79 & 0.60 & 1.05 \\
\hline & Bolton Teaching & 266,500 & 390 & 432 & 439 & 454 & 507 & 1.22 & 1.03 & 1.45 \\
\hline & Bury & 183,500 & 360 & 349 & 409 & 409 & 420 & 0.99 & 0.79 & 1.24 \\
\hline & Central and Eastern Cheshire & 457,200 & 302 & 304 & 306 & 341 & 361 & 0.81 & 0.69 & 0.94 \\
\hline & Central Lancashire & 459,200 & 296 & 318 & 329 & 359 & 388 & 0.90 & 0.78 & 1.04 \\
\hline & Cumbria Teaching & 494,400 & 316 & 332 & 372 & 394 & 394 & 0.86 & 0.75 & 0.99 \\
\hline & East Lancashire Teaching & 381,200 & 399 & 412 & 409 & 407 & 438 & 1.03 & 0.88 & 1.20 \\
\hline & Halton and St Helens & 296,700 & 283 & 310 & 327 & 361 & 381 & 0.88 & 0.73 & 1.06 \\
\hline & Heywood, Middleton and Rochdale & 205,000 & 390 & 405 & 420 & 429 & 468 & 1.14 & 0.93 & 1.39 \\
\hline & Knowsley & 149,200 & 308 & 315 & 342 & 355 & 342 & 0.83 & 0.63 & 1.09 \\
\hline & Liverpool & 445,300 & 310 & 332 & 350 & 375 & 409 & 1.03 & 0.89 & 1.19 \\
\hline & Manchester Teaching & 498,800 & 233 & 247 & 249 & 297 & 333 & 0.95 & 0.81 & 1.10 \\
\hline & North Lancashire Teaching & 329,100 & 319 & 313 & 310 & 304 & 310 & 0.71 & 0.59 & 0.86 \\
\hline & Oldham & 219,600 & 351 & 369 & 387 & 410 & 414 & 1.02 & 0.83 & 1.25 \\
\hline & Salford & 229,100 & 266 & 306 & 327 & 362 & 388 & 0.97 & 0.79 & 1.20 \\
\hline & Sefton & 272,800 & 323 & 301 & 319 & 356 & 363 & 0.83 & 0.68 & 1.01 \\
\hline & Stockport & 284,700 & 330 & 351 & 376 & 400 & 418 & 0.96 & 0.80 & 1.15 \\
\hline & Tameside and Glossop & 250,700 & 415 & 415 & 423 & 459 & 503 & 1.18 & 0.99 & 1.40 \\
\hline & Trafford & 217,100 & 290 & 309 & 299 & 336 & 359 & 0.85 & 0.68 & 1.06 \\
\hline & Warrington & 199,100 & 387 & 387 & 417 & 387 & 402 & 0.92 & 0.74 & 1.14 \\
\hline & Western Cheshire & 234,300 & 333 & 324 & 367 & 393 & 410 & 0.94 & 0.77 & 1.14 \\
\hline & Wirral & 308,800 & 298 & 324 & 340 & 350 & 353 & 0.83 & 0.68 & 1.00 \\
\hline \multirow{6}{*}{$\begin{array}{l}\text { Yorkshire and the } \\
\text { Humber }\end{array}$} & Barnsley & 227,500 & 347 & 374 & 378 & 400 & 413 & 0.95 & 0.77 & 1.16 \\
\hline & Bradford and Airedale Teaching & 512,700 & 363 & 392 & 419 & 447 & 453 & 1.18 & 1.04 & 1.34 \\
\hline & Calderdale & 202,800 & 414 & 454 & 464 & 498 & 533 & 1.24 & 1.03 & 1.50 \\
\hline & Doncaster & 290,900 & 313 & 333 & 358 & 364 & 395 & 0.92 & 0.77 & 1.10 \\
\hline & East Riding of Yorkshire & 338,500 & 301 & 331 & 357 & 369 & 381 & 0.83 & 0.70 & 0.99 \\
\hline & Hull Teaching & 263,800 & 322 & 341 & 364 & 371 & 394 & 0.98 & 0.81 & 1.19 \\
\hline
\end{tabular}


Table 3.4. Continued

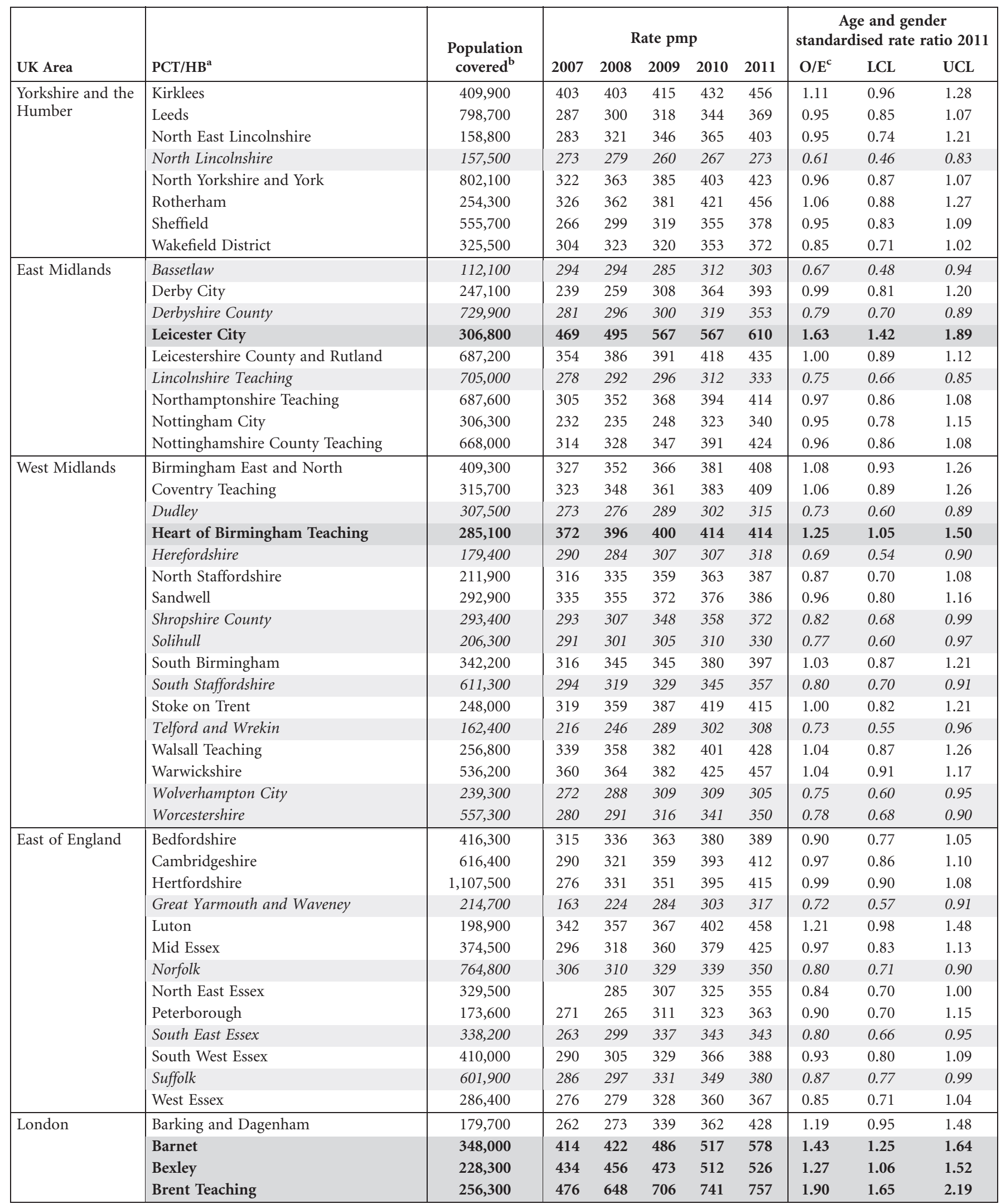


Table 3.4. Continued

\begin{tabular}{|c|c|c|c|c|c|c|c|c|c|c|}
\hline \multirow[b]{2}{*}{ UK Area } & \multirow[b]{2}{*}{$\mathrm{PCT} / \mathrm{HB}^{\mathrm{a}}$} & \multirow{2}{*}{$\begin{array}{l}\text { Population } \\
\text { covered }^{\mathbf{b}}\end{array}$} & \multicolumn{5}{|c|}{ Rate pmp } & \multicolumn{3}{|c|}{$\begin{array}{c}\text { Age and gender } \\
\text { standardised rate ratio } 2011\end{array}$} \\
\hline & & & 2007 & 2008 & 2009 & 2010 & 2011 & $\mathrm{O} / \mathrm{E}^{\mathrm{c}}$ & LCL & UCL \\
\hline \multirow[t]{27}{*}{ London } & Bromley & 312,400 & 416 & 435 & 448 & 483 & 493 & 1.17 & 1.00 & 1.37 \\
\hline & Camden & 235,500 & 276 & 344 & 386 & 408 & 454 & 1.17 & 0.97 & 1.42 \\
\hline & City and Hackney Teaching & 231,000 & 277 & 312 & 338 & 359 & 359 & 0.96 & 0.78 & 1.19 \\
\hline & Croydon & 345,400 & 310 & 327 & 368 & 379 & 411 & 1.00 & 0.85 & 1.18 \\
\hline & Ealing & 318,300 & 437 & 566 & 587 & 631 & 653 & 1.62 & 1.41 & 1.85 \\
\hline & Enfield & 295,000 & 414 & 461 & 468 & 505 & 573 & 1.43 & 1.23 & 1.66 \\
\hline & Greenwich Teaching & 228,100 & 307 & 333 & 395 & 438 & 469 & 1.22 & 1.01 & 1.47 \\
\hline & Hammersmith and Fulham & 169,800 & 283 & 347 & 436 & 471 & 477 & 1.22 & 0.98 & 1.51 \\
\hline & Haringey Teaching & 225,100 & 360 & 413 & 466 & 511 & 555 & 1.40 & 1.17 & 1.66 \\
\hline & Harrow & 230,300 & 456 & 595 & 664 & 725 & 738 & 1.79 & 1.54 & 2.08 \\
\hline & Havering & 236,100 & 263 & 280 & 305 & 313 & 335 & 0.79 & 0.64 & 0.99 \\
\hline & Hillingdon & 266,200 & 334 & 432 & 488 & 526 & 575 & 1.45 & 1.24 & 1.70 \\
\hline & Hounslow & 236,700 & 342 & 444 & 515 & 566 & 575 & 1.43 & 1.21 & 1.69 \\
\hline & Islington & 193,900 & 397 & 433 & 474 & 511 & 536 & 1.39 & 1.15 & 1.69 \\
\hline & Kensington and Chelsea & 169,500 & 277 & 342 & 360 & 431 & 448 & 1.06 & 0.85 & 1.33 \\
\hline & Kingston & 169,000 & 349 & 373 & 391 & 396 & 414 & 1.03 & 0.81 & 1.30 \\
\hline & Lambeth & 284,400 & 281 & 316 & 359 & 359 & 394 & 1.01 & 0.84 & 1.21 \\
\hline & Lewisham & 266,400 & 402 & 398 & 420 & 439 & 458 & 1.15 & 0.96 & 1.37 \\
\hline & Newham & 240,200 & 287 & 316 & 387 & 441 & 466 & 1.31 & 1.09 & 1.58 \\
\hline & Redbridge & 270,300 & 314 & 363 & 392 & 474 & 499 & 1.27 & 1.07 & 1.50 \\
\hline & Richmond and Twickenham & 190,800 & 204 & 257 & 294 & 309 & 341 & 0.80 & 0.63 & 1.02 \\
\hline & Southwark & 287,100 & 401 & 404 & 460 & 491 & 526 & 1.35 & 1.15 & 1.58 \\
\hline & Sutton and Merton & 403,000 & 362 & 375 & 409 & 427 & 442 & 1.08 & 0.93 & 1.25 \\
\hline & Tower Hamlets & 238,100 & 235 & 231 & 265 & 315 & 323 & 0.92 & 0.74 & 1.15 \\
\hline & Waltham Forest & 227,400 & 378 & 405 & 431 & 475 & 510 & 1.32 & 1.10 & 1.59 \\
\hline & Wandsworth & 289,200 & 342 & 349 & 353 & 373 & 422 & 1.10 & 0.92 & 1.31 \\
\hline & Westminster & 253,400 & 233 & 320 & 395 & 430 & 430 & 1.06 & 0.88 & 1.28 \\
\hline \multirow[t]{8}{*}{ South East Coast } & Brighton and Hove City & 258,400 & 267 & 290 & 313 & 344 & 364 & 0.91 & 0.74 & 1.11 \\
\hline & East Sussex Downs and Weald & 336,100 & 271 & 301 & 318 & 327 & 342 & 0.78 & 0.65 & 0.93 \\
\hline & Eastern and Coastal Kent & 742,200 & 298 & 346 & 380 & 406 & 441 & 1.04 & 0.93 & 1.16 \\
\hline & Hastings and Rother & 179,700 & 295 & 312 & 312 & 328 & 351 & 0.79 & 0.62 & 1.01 \\
\hline & Medway & 256,600 & 316 & 378 & 413 & 417 & 429 & 1.02 & 0.85 & 1.24 \\
\hline & Surrey & $1,114,400$ & 337 & 354 & 371 & 386 & 391 & 0.91 & 0.83 & 1.00 \\
\hline & West Kent & 685,100 & 343 & 371 & 401 & 404 & 410 & 0.95 & 0.85 & 1.07 \\
\hline & West Sussex & 800,000 & 318 & 338 & 345 & 364 & 381 & 0.88 & 0.78 & 0.98 \\
\hline \multirow[t]{9}{*}{ South Central } & Berkshire East & 406,500 & 364 & 408 & 445 & 504 & 526 & 1.29 & 1.13 & 1.48 \\
\hline & Berkshire West & 471,500 & 375 & 409 & 445 & 454 & 477 & 1.15 & 1.01 & 1.31 \\
\hline & Buckinghamshire & 512,100 & 414 & 420 & 426 & 453 & 467 & 1.08 & 0.96 & 1.23 \\
\hline & Hampshire & $1,297,200$ & 325 & 358 & 373 & 392 & 405 & 0.93 & 0.85 & 1.01 \\
\hline & Isle of Wight National Health Service & 140,200 & 257 & 307 & 321 & 335 & 335 & 0.74 & 0.55 & 0.98 \\
\hline & Milton Keynes & 247,000 & 312 & 332 & 352 & 393 & 429 & 1.03 & 0.85 & 1.24 \\
\hline & Oxfordshire & 624,200 & 394 & 409 & 413 & 433 & 449 & 1.09 & 0.97 & 1.22 \\
\hline & Portsmouth City Teaching & 207,200 & 333 & 362 & 362 & 405 & 401 & 1.05 & 0.85 & 1.30 \\
\hline & Southampton City & 239,800 & 334 & 342 & 354 & 350 & 396 & 1.06 & 0.86 & 1.29 \\
\hline \multirow[t]{7}{*}{ South West } & Bath and North East Somerset & 179,800 & 284 & 289 & 323 & 311 & 306 & 0.75 & 0.58 & 0.98 \\
\hline & Bournemouth and Poole Teaching & 310,800 & 357 & 347 & 344 & 354 & 376 & 0.91 & 0.76 & 1.09 \\
\hline & Bristol & 441,100 & 385 & 419 & 431 & 460 & 472 & 1.23 & 1.07 & 1.41 \\
\hline & Cornwall and Isles of Scilly & 537,900 & 368 & 405 & 431 & 441 & 465 & 1.04 & 0.92 & 1.17 \\
\hline & Devon & 749,700 & 328 & 351 & 384 & 400 & 403 & 0.90 & 0.81 & 1.01 \\
\hline & Dorset & 404,900 & 403 & 427 & 437 & 454 & 452 & 1.00 & 0.86 & 1.16 \\
\hline & Gloucestershire & 593,600 & 322 & 334 & 335 & 345 & 382 & 0.88 & 0.77 & 1.00 \\
\hline
\end{tabular}


Table 3.4. Continued

\begin{tabular}{|c|c|c|c|c|c|c|c|c|c|c|}
\hline \multirow[b]{2}{*}{ UK Area } & \multirow[b]{2}{*}{$\mathrm{PCT} / \mathrm{HB}^{\mathrm{a}}$} & \multirow{2}{*}{$\begin{array}{l}\text { Population } \\
\text { covered }^{\text {b }}\end{array}$} & \multicolumn{5}{|c|}{ Rate pmp } & \multicolumn{3}{|c|}{$\begin{array}{c}\text { Age and gender } \\
\text { standardised rate ratio } 2011\end{array}$} \\
\hline & & & 2007 & 2008 & 2009 & 2010 & 2011 & $\mathrm{O} / \mathrm{E}^{\mathrm{c}}$ & LCL & UCL \\
\hline \multirow[t]{7}{*}{ South West } & North Somerset & 212,100 & 344 & 368 & 391 & 415 & 424 & 0.96 & 0.78 & 1.18 \\
\hline & Plymouth Teaching & 258,900 & 417 & 463 & 498 & 506 & 537 & 1.35 & 1.14 & 1.59 \\
\hline & Somerset & 525,500 & 352 & 352 & 371 & 390 & 424 & 0.96 & 0.84 & 1.09 \\
\hline & South Gloucestershire & 264,900 & 430 & 438 & 442 & 464 & 479 & 1.12 & 0.94 & 1.34 \\
\hline & Swindon & 206,900 & 314 & 348 & 358 & 420 & 440 & 1.04 & 0.85 & 1.28 \\
\hline & Torbay & 134,400 & 335 & 394 & 446 & 469 & 491 & 1.11 & 0.87 & 1.42 \\
\hline & Wiltshire & 459,800 & 296 & 313 & 318 & 352 & 381 & 0.87 & 0.75 & 1.01 \\
\hline \multirow[t]{7}{*}{ Wales } & Betsi Cadwaladr University & 678,500 & 314 & 333 & 343 & 355 & 368 & 0.84 & 0.74 & 0.95 \\
\hline & Powys Teaching & 131,100 & 336 & 359 & 374 & 412 & 404 & 0.87 & 0.67 & 1.14 \\
\hline & Hywel Dda & 374,800 & 358 & 382 & 398 & 398 & 424 & 0.96 & 0.82 & 1.12 \\
\hline & Abertawe Bro Morgannwg University & 504,800 & 428 & 442 & 468 & 501 & 563 & 1.32 & 1.17 & 1.48 \\
\hline & Cwm Taf & 290,600 & 516 & 544 & 578 & 643 & 678 & 1.61 & 1.40 & 1.85 \\
\hline & Aneurin Bevan & 561,300 & 433 & 451 & 472 & 515 & 534 & 1.25 & 1.12 & 1.40 \\
\hline & Cardiff and Vale University & 466,100 & 390 & 408 & 414 & 446 & 478 & 1.22 & 1.07 & 1.39 \\
\hline \multirow[t]{14}{*}{ Scotland } & Ayrshire \& Arran & 366,900 & 376 & 403 & 398 & 395 & 395 & 0.88 & 0.75 & 1.04 \\
\hline & Borders & 113,000 & 310 & 363 & 372 & 434 & 434 & 0.94 & 0.71 & 1.24 \\
\hline & Dumfries and Galloway & 148,100 & 344 & 371 & 392 & 392 & 412 & 0.89 & 0.69 & 1.14 \\
\hline & Fife & 364,800 & 296 & 318 & 326 & 345 & 370 & 0.85 & 0.72 & 1.01 \\
\hline & Forth Valley & 293,100 & 297 & 307 & 304 & 324 & 348 & 0.80 & 0.66 & 0.97 \\
\hline & Grampian & 550,500 & 345 & 358 & 391 & 407 & 420 & 0.96 & 0.84 & 1.09 \\
\hline & Greater Glasgow \& Clyde & $1,204,100$ & 409 & 428 & 434 & 445 & 462 & 1.09 & 1.01 & 1.19 \\
\hline & Highland & 310,700 & 380 & 435 & 489 & 518 & 515 & 1.12 & 0.96 & 1.30 \\
\hline & Lanarkshire & 562,700 & 370 & 389 & 411 & 423 & 448 & 1.04 & 0.92 & 1.17 \\
\hline & Lothian & 837,000 & 307 & 327 & 338 & 356 & 375 & 0.89 & 0.80 & 1.00 \\
\hline & Orkney & 19,800 & 455 & 556 & 455 & 404 & 404 & 0.86 & 0.43 & 1.73 \\
\hline & Shetland & 22,500 & 267 & 222 & 267 & 267 & 222 & 0.49 & 0.21 & 1.19 \\
\hline & Tayside & 402,400 & 417 & 432 & 430 & 432 & 440 & 1.02 & 0.88 & 1.18 \\
\hline & Western Isles & 26,500 & 302 & 302 & 302 & 302 & 302 & 0.65 & 0.33 & 1.30 \\
\hline \multirow[t]{5}{*}{ Northern Ireland } & Belfast & 335,700 & 375 & 378 & 399 & 441 & 450 & 1.15 & 0.98 & 1.35 \\
\hline & Northern & 458,600 & 325 & 347 & 360 & 375 & 392 & 0.95 & 0.82 & 1.10 \\
\hline & Southern & 357,700 & 296 & 296 & 299 & 319 & 358 & 0.91 & 0.77 & 1.09 \\
\hline & South Eastern & 347,100 & 340 & 354 & 363 & 366 & 398 & 0.95 & 0.81 & 1.13 \\
\hline & Western & 299,900 & 293 & 303 & 320 & 340 & 357 & 0.89 & 0.74 & 1.08 \\
\hline
\end{tabular}

Primary renal diagnosis

The overall proportion of patients with a PRD of glomerulonephritis was slightly higher than that reported in previous reports as a consequence of reclassifying 'glomerulonephritis biopsy unproven' this year (as discussed in methods). This change in methodology notwithstanding the primary renal diagnosis of patients receiving kidney transplants in the UK has remained relatively stable over the last five years (table 3.7).

\section{Ethnicity}

It was difficult to compare the proportion of patients within each ethnic group receiving a transplant to those commencing dialysis from the same group because data on ethnicity were missing in a considerable number of patients who were classified as ethnicity 'unknown' (table 3.8). The percentages of patients with unknown ethnicity between 2006 and 2010 provided in this year's chapter are different from those in last year's chapter [2]; this reflects retrospective input of ethnicity data, improving data completeness.

\section{Clinical and laboratory outcomes}

\section{Introduction}

There continued to be marked variation in the completeness of data (tables 3.9a, 3.9b) reported by each renal centre, particularly for blood pressure. Better data records (or possibly better extraction of data held within 
Table 3.5. Distribution of prevalent patients on RRT by centre and modality on 31/12/2011

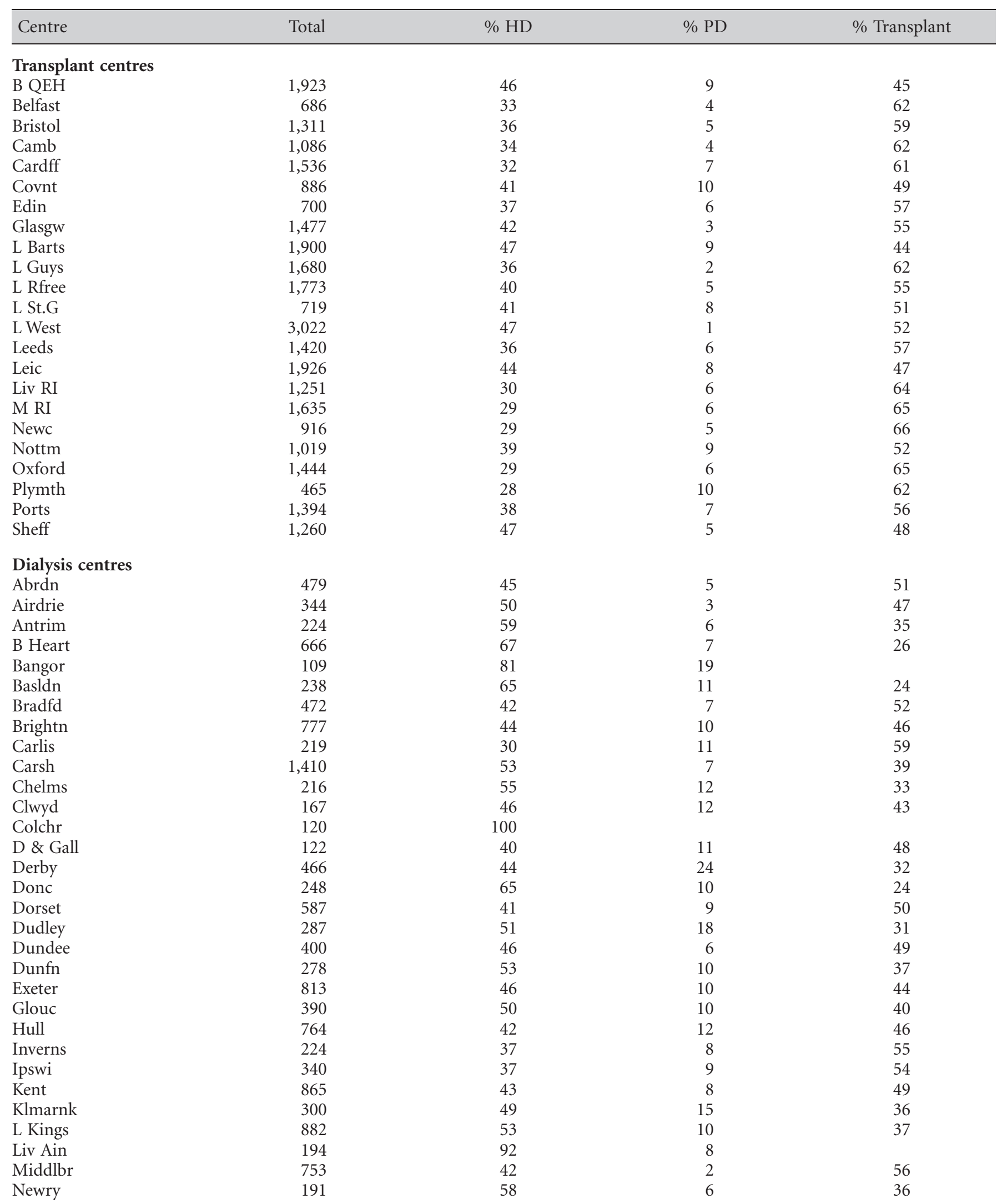


Table 3.5. Continued

\begin{tabular}{|c|c|c|c|c|}
\hline Centre & Total & $\% \mathrm{HD}$ & $\% \mathrm{PD}$ & $\%$ Transplant \\
\hline Norwch & 612 & 50 & 10 & 40 \\
\hline Prestn & 1,023 & 51 & 6 & 43 \\
\hline Salford & 846 & 43 & 13 & 44 \\
\hline Shrew & 342 & 55 & 10 & 35 \\
\hline Stevng & 638 & 65 & 5 & 31 \\
\hline Sund & 390 & 46 & 4 & 50 \\
\hline Swanse & 659 & 54 & 9 & 37 \\
\hline Truro & 357 & 43 & 7 & 50 \\
\hline Ulster & 137 & 77 & 2 & 21 \\
\hline West NI & 272 & 55 & 7 & 38 \\
\hline Wirral & 241 & 81 & 17 & 1 \\
\hline England & 44,665 & 43 & 7 & 49 \\
\hline N Ireland & 1,510 & 48 & 5 & 47 \\
\hline Scotland & 4,324 & 43 & 6 & 51 \\
\hline Wales & 2,708 & 41 & 8 & 51 \\
\hline UK & 53,207 & 43 & 7 & 49 \\
\hline
\end{tabular}

Table 3.6. Median age and gender ratio of incident and prevalent transplant patients 2006-2011

\begin{tabular}{|c|c|c|c|c|c|c|}
\hline Year & \multicolumn{3}{|c|}{ Incident transplants } & \multicolumn{3}{|c|}{ Prevalent transplants ${ }^{\star}$} \\
\hline 2007 & 2,118 & 45.6 & 1.6 & 20,793 & 50.2 & 1.5 \\
\hline 2008 & 2,337 & 46.4 & 1.5 & 22,281 & 50.4 & 1.5 \\
\hline 2009 & 2,481 & 48.4 & 1.6 & 23,534 & 50.7 & 1.5 \\
\hline
\end{tabular}

${ }^{*}$ As on 31 st December for given year

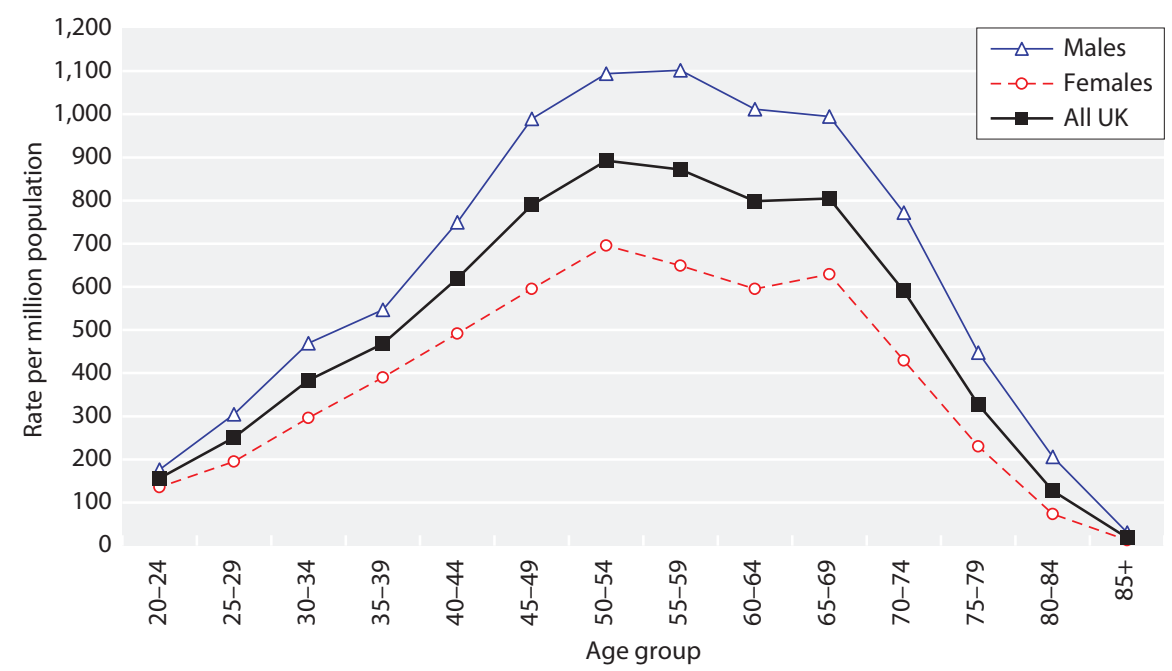

Fig. 3.1. Transplant prevalence rate per million population by age and gender on $31 / 12 / 2011$

Pruthi/Casula/MacPhee 
Table 3.7. Primary renal diagnosis in renal transplant recipients 2006-2011

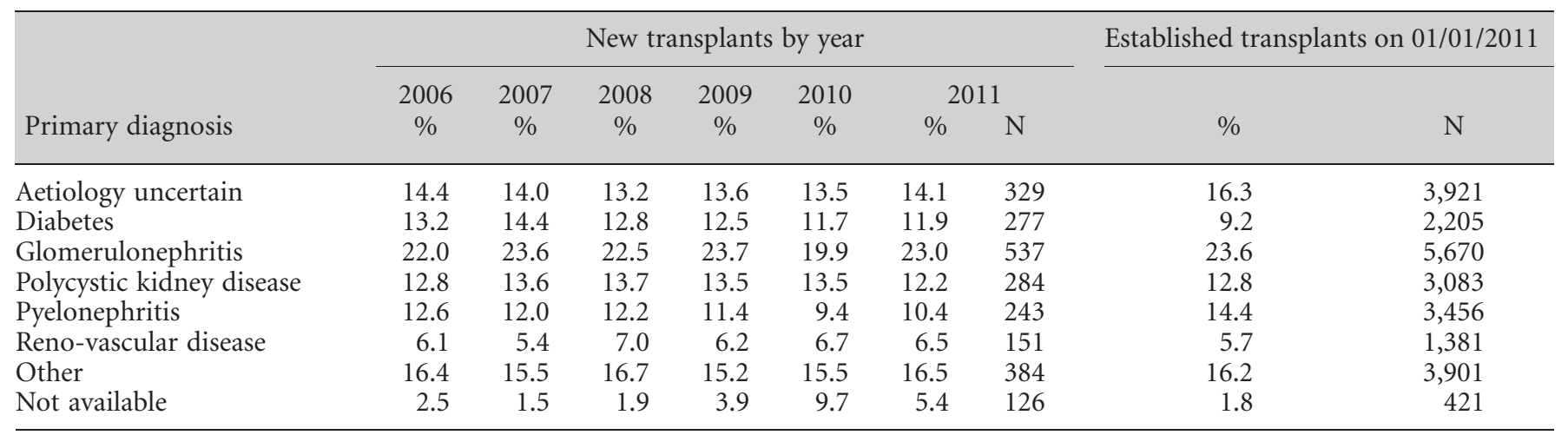

renal IT systems) would facilitate more meaningful comparisons between centres and help to determine the causes of inter-centre differences in outcomes. For this reason, along with differences in repatriation policies of prevalent transplant patients between centres as highlighted previously, caution needs to be exercised when comparing centre performance.

The 71 renal centres in the UK comprise 52 centres in England, 5 in Wales, 5 in Northern Ireland and 9 in Scotland. Centres in Scotland only provide summary information and therefore laboratory outcome data for comparisons were not available for the Scottish renal centres. Three centres (Bangor, Colchester, Liverpool Aintree) were reported as having no transplanted patients and were therefore excluded. After exclusion of these 12 centres, prevalent patient data from 59 renal centres across the UK were analysed.

For the one year post-transplant analyses, in which patients were assigned to the centres that performed their transplant, the two Scottish transplant centres were excluded as they only submit limited biochemical data to the UKRR. After excluding these 2 transplant centres, one year outcomes are described for 21 transplant centres across the UK.

\section{Methods}

Data for key laboratory variables are reported for all prevalent patients with valid data returns for a given renal centre (both transplanting and non-transplanting centres) and for one year post-transplant results for patients transplanted 2004-2010, with patients attributed to the transplant centre that performed the procedure.

Time since transplantation may have a significant effect on key biochemical and clinical variables and this is likely to be independent of a centre's clinical practices. Therefore, inter-centre comparison of data on prevalent transplant patients is open to bias. To minimise bias relating to fluctuations in biochemical and clinical parameters occurring in the initial post-transplant period, one year post-transplantation outcomes are also reported. It is presumed that patient selection policies and local clinical practices are more likely to be relevant in influencing outcomes 12 months post-transplant and therefore comparison of outcomes between centres is more robust. However, even the 12 months posttransplant comparisons could be biased by the fact that in some centres, repatriation of patients only occurs if the graft is failing whereas in others it only occurs if the graft function is stable.

Centres with $<20$ patients or $<50 \%$ data completeness have been excluded from the figures.

\section{Prevalent patient data}

Biochemical and clinical data for patients with a functioning transplant followed in either a transplanting or non-transplanting centre were included in the analyses. The cohort consisted of prevalent patients as on 31st December 2011. Patients were considered as having a functioning transplant if 'transplant' was listed as the last mode of RRT in the last quarter of 2011. Patients were assigned to the renal centre that sent the data to the UKRR but some patients will have received care in more than one centre. If data for the same transplant patient were received from both the transplant centre and non-transplant centre, care was allocated to the non-transplant centre. Patients with a functioning transplant

Table 3.8. Ethnicity of patients who received a transplant in the years 2006-2011

\begin{tabular}{lccccr}
\hline Year & \% White & \% South Asian & \% African Caribbean & \% Other & \% Unknown \\
\hline 2006 & 75.5 & 8.2 & 6.4 & 2.0 & 7.9 \\
2007 & 75.6 & 7.9 & 5.9 & 6.0 & 1.8 \\
2008 & 72.7 & 8.6 & 6.2 & 2.3 & 2.7 \\
2009 & 71.4 & 10.1 & 6.7 & 6.1 & 9.5 \\
2010 & 72.3 & 10.0 & 6.6 & 2.1 & 9.2 \\
2011 & 72.6 & 9.3 & & \\
\hline
\end{tabular}


Table 3.9a. Percentage completeness by centre for prevalent transplant patients on 31/12/2011 ${ }^{\mathrm{a}}$

\begin{tabular}{|c|c|c|c|c|c|c|c|c|c|}
\hline Centre & $\mathrm{N}$ & Ethnicity & $\mathrm{eGFR}^{\mathrm{b}}$ & $\begin{array}{c}\text { Blood } \\
\text { pressure }\end{array}$ & Centre & $\mathrm{N}$ & Ethnicity & eGFR ${ }^{b}$ & $\begin{array}{c}\text { Blood } \\
\text { pressure }\end{array}$ \\
\hline England & & & & & Norwch & 239 & 97 & 97 & 47 \\
\hline B QEH & 834 & 100 & 94 & 93 & Oxford & 902 & 92 & 99 & 16 \\
\hline Basldn & 55 & 100 & 98 & 13 & Plymth & 274 & 99 & 95 & 0 \\
\hline Bradfd & 237 & 98 & 87 & 74 & Ports & 754 & 99 & 96 & 12 \\
\hline Camb & 626 & 98 & 100 & 98 & Salford & 364 & 99 & 95 & 0 \\
\hline Carlis & 128 & 98 & 96 & 0 & Sheff & 594 & 100 & 99 & 96 \\
\hline Carsh & 541 & 96 & 90 & 0 & Shrew & 117 & 100 & 53 & 0 \\
\hline Chelms & 67 & 99 & 97 & 87 & Stevng & 192 & 100 & 69 & 35 \\
\hline Covnt & 417 & 99 & 90 & 51 & Sthend & 73 & 100 & 100 & 56 \\
\hline Derby & 133 & 99 & 98 & 82 & Stoke & 289 & 59 & 99 & 0 \\
\hline Glouc & 154 & 100 & 97 & 88 & York & 166 & 80 & 99 & 42 \\
\hline Hull & 335 & 62 & 95 & 0 & N Ireland & & & & \\
\hline Ipswi & 178 & 99 & 99 & 85 & Antrim & 77 & 100 & 97 & 91 \\
\hline Kent & 392 & 95 & 49 & 87 & Belfast & 415 & 100 & 99 & 47 \\
\hline L Barts & 804 & 100 & 97 & 0 & Newry & 67 & 100 & 94 & 90 \\
\hline L Guys & 1,001 & 81 & 97 & 0 & Ulster & 25 & 100 & 96 & 84 \\
\hline L Kings & 315 & 98 & 96 & 0 & West NI & 101 & 100 & 96 & 89 \\
\hline L RFree & 928 & 98 & 94 & 0 & Wales & & & & \\
\hline L St.G & 358 & 88 & 95 & 1 & Cardff & 910 & 75 & 99 & 97 \\
\hline L West & 1,542 & 100 & 97 & 0 & Clwyd & 64 & 80 & 94 & 86 \\
\hline Leeds & 814 & 90 & 97 & 96 & Swanse & 230 & 99 & 97 & 99 \\
\hline
\end{tabular}

${ }^{a}$ Scottish centres not shown as a limited dataset was returned that could not be included for technical reasons

${ }^{\mathrm{b}}$ Patients with missing ethnicity were classed as White for eGFR calculation

${ }^{\mathrm{c}}$ Data relating to blood pressure could not be extracted from these centres due to technical problems

of less than three months duration were excluded from analyses. For haemoglobin, estimated glomerular filtration rate (eGFR), corrected calcium, phosphate and blood pressure (BP), the latest value in quarter 3 or quarter 4 of 2011 was used.

\section{Estimated glomerular filtration rate (eGFR)}

For the purpose of eGFR calculation, the original 4-variable MDRD formula was used (with a constant of 186) to calculate eGFR from the serum creatinine concentration as reported by the centre (unless otherwise stated). A wide variety of creatinine assays are in use in clinical biochemistry laboratories in the UK, and it is not possible to ensure that all measurements of creatinine concentration collected by the UKRR are harmonised. Although many laboratories are now reporting assay results that have been aligned to the isotope dilution-mass spectrometry standard (which would necessitate use of the modified MDRD formula), this was not the case at the end of 2011. Patients with valid serum creatinine results but no ethnicity data were classed as White for the purpose of the eGFR calculation.

\section{One year post-transplant data}

Patients who received a renal transplant between 1st January 2004 and 31st December 2010 were assigned according to the renal centre in which they were transplanted. In a small number of instances, the first documented evidence of transplantation in a patient's record is from a timeline entry in data returned from a non-transplant centre, in these instances the patient was reassigned to the nearest transplant centre (table 3.10).

Patients who had died or experienced graft failure within 12 months of transplantation were excluded from the analyses. Patients with more than one transplant during 2004-2010 were included as separate episodes provided each of the transplants functioned for a year

For each patient, the most recent laboratory or blood pressure 
Table 3.9b. Percentage completeness by centre for prevalent transplant patients on 31/12/2011

\begin{tabular}{|c|c|c|c|c|c|c|}
\hline Centre & $\mathrm{N}$ & Haemoglobin & $\begin{array}{c}\text { Total serum } \\
\text { cholesterol }\end{array}$ & $\begin{array}{l}\text { Adjusted serum } \\
\text { calcium }^{\mathrm{b}}\end{array}$ & $\begin{array}{c}\text { Serum } \\
\text { phosphate }\end{array}$ & $\begin{array}{c}\text { Serum } \\
\text { PTH }\end{array}$ \\
\hline \multicolumn{7}{|l|}{ England } \\
\hline B Heart & 162 & 93 & 41 & 86 & 86 & 13 \\
\hline B QEH & 834 & 94 & 72 & 94 & 93 & 62 \\
\hline Basldn & 55 & 95 & 44 & 96 & 85 & 53 \\
\hline Bradfd & 237 & 79 & 43 & 85 & 83 & 27 \\
\hline Brightn & 346 & 88 & 23 & 73 & 84 & 25 \\
\hline Bristol & 745 & 99 & 70 & 99 & 99 & 98 \\
\hline Camb & 626 & 99 & 72 & 99 & 99 & 89 \\
\hline Carlis & 128 & 95 & 65 & 92 & 92 & 14 \\
\hline Carsh & 541 & 71 & 51 & 89 & 89 & 0 \\
\hline Chelms & 67 & 97 & 66 & 97 & 97 & 22 \\
\hline Covnt & 417 & 89 & 0 & 89 & 64 & 26 \\
\hline Derby & 133 & 94 & 58 & 93 & 89 & 77 \\
\hline Donc & 60 & 100 & 85 & 100 & 100 & 32 \\
\hline Dorset & 285 & 89 & 53 & 55 & 51 & 20 \\
\hline Dudley & 85 & 98 & 61 & 69 & 98 & 59 \\
\hline Exeter & 345 & 97 & 91 & 97 & 94 & 14 \\
\hline Glouc & 154 & 97 & 39 & 96 & 94 & 31 \\
\hline Hull & 335 & 94 & 24 & 92 & 92 & 18 \\
\hline Ipswi & 178 & 99 & 30 & 99 & 99 & 58 \\
\hline Kent & 392 & 96 & 52 & 93 & 93 & 7 \\
\hline L Barts & 804 & 97 & 96 & 94 & 94 & 69 \\
\hline L Guys & 1,001 & 97 & 31 & 92 & 92 & 31 \\
\hline L Kings & 315 & 96 & 41 & 96 & 96 & 20 \\
\hline L RFree & 928 & 61 & 74 & 94 & 94 & 57 \\
\hline L St.G & 358 & 94 & 40 & 95 & 95 & 46 \\
\hline L West & 1,542 & 98 & 26 & 98 & 98 & 7 \\
\hline Leeds & 814 & 96 & 91 & 96 & 96 & 48 \\
\hline Leic & 877 & 94 & 89 & 94 & 94 & 58 \\
\hline Liv RI & 775 & 89 & 3 & 87 & 88 & 71 \\
\hline M RI & 1,020 & 99 & 49 & 99 & 99 & 60 \\
\hline Middlbr & 414 & 95 & 41 & 93 & 92 & 12 \\
\hline Newc & 587 & 99 & 72 & 97 & 99 & 37 \\
\hline Norwch & 239 & 97 & 94 & 96 & 96 & 29 \\
\hline Nottm & 506 & 100 & 60 & 96 & 95 & 83 \\
\hline Oxford & 902 & 99 & 52 & 99 & 99 & 27 \\
\hline Plymth & 274 & 83 & 42 & 91 & 89 & 20 \\
\hline Ports & 754 & 95 & 32 & 94 & 89 & 13 \\
\hline Prestn & 432 & 96 & 47 & 93 & 91 & 38 \\
\hline Redng & 299 & 99 & 78 & 99 & 85 & 59 \\
\hline Salford & 364 & 95 & 82 & 95 & 95 & 82 \\
\hline Sheff & 594 & 99 & 39 & 98 & 98 & 22 \\
\hline Shrew & 117 & 85 & 71 & 77 & 76 & 5 \\
\hline Stevng & 192 & 96 & 73 & 95 & 92 & 45 \\
\hline Sthend & 73 & 99 & 30 & 99 & 95 & 8 \\
\hline Stoke & 289 & 99 & 97 & 99 & 99 & 28 \\
\hline Sund & 188 & 99 & 88 & 99 & 99 & 86 \\
\hline Truro & 170 & 97 & 49 & 97 & 97 & 40 \\
\hline Wirral & 3 & 100 & 100 & 33 & 100 & 67 \\
\hline Wolve & 136 & 97 & 55 & 97 & 90 & 46 \\
\hline York & 166 & 87 & 61 & 85 & 96 & 16 \\
\hline
\end{tabular}


Table 3.9b. Continued

\begin{tabular}{|c|c|c|c|c|c|c|}
\hline Centre & $\mathrm{N}$ & Haemoglobin & $\begin{array}{l}\text { Total serum } \\
\text { cholesterol }\end{array}$ & $\begin{array}{l}\text { Adjusted serum } \\
\text { calcium }^{\mathrm{b}}\end{array}$ & $\begin{array}{c}\text { Serum } \\
\text { phosphate }\end{array}$ & $\begin{array}{c}\text { Serum } \\
\text { PTH }\end{array}$ \\
\hline \multicolumn{7}{|l|}{$\mathrm{N}$ Ireland } \\
\hline Antrim & 77 & 96 & 94 & 95 & 96 & 94 \\
\hline Belfast & 415 & 99 & 98 & 97 & 97 & 25 \\
\hline Newry & 67 & 94 & 40 & 94 & 94 & 63 \\
\hline Ulster & 25 & 96 & 96 & 96 & 96 & 68 \\
\hline West NI & 101 & 97 & 92 & 94 & 94 & 69 \\
\hline \multicolumn{7}{|l|}{ Wales } \\
\hline Cardff & 910 & 99 & 51 & 99 & 98 & 15 \\
\hline Clwyd & 64 & 92 & 86 & 94 & 94 & 53 \\
\hline Swanse & 230 & 97 & 72 & 97 & 97 & 43 \\
\hline Wrexm & 128 & 98 & 90 & 97 & 97 & 99 \\
\hline England & 21,258 & 94 & 55 & 94 & 93 & 42 \\
\hline $\mathrm{N}$ Ireland & 685 & 98 & 91 & 96 & 96 & 45 \\
\hline Wales & 1,332 & 98 & 60 & 98 & 98 & 30 \\
\hline E, W \& NI & 23,275 & 94 & 56 & 94 & 93 & 41 \\
\hline
\end{tabular}

${ }^{a}$ Scottish centres not shown as a limited dataset was returned that could not be included for technical reasons

${ }^{\mathrm{b}}$ Serum calcium corrected for serum albumin

for the relevant 4 th/5th quarter (10-15 months) after renal transplantation was taken to be representative of the one year post-transplant outcome. Again, for the purpose of the eGFR calculation patients with valid serum creatinine results but missing ethnicity data were classed as White.

\section{Results and discussion}

Post-transplant eGFR in prevalent transplant patients

When interpreting eGFR post-transplantation, it is important to remember that estimated GFR formulae

Table 3.10. Number of patients per transplant centre after allocation of patients in non-transplant centres ${ }^{*}$ (transplanted between 2004-2010)

\begin{tabular}{|c|c|c|c|}
\hline Transplant centre & $\begin{array}{l}\text { Total number of patients } \\
\text { per transplant centre }\end{array}$ & Non-transplant centre & $\begin{array}{l}\text { Number of patients reallocated } \\
\text { to a transplant centre }\end{array}$ \\
\hline B QEH & 848 & Stoke & 4 \\
\hline \multirow[t]{3}{*}{ Belfast } & 261 & Antrim & 2 \\
\hline & & Newry & 7 \\
\hline & & West NI & 4 \\
\hline Bristol & 684 & Dorset & 1 \\
\hline Camb & 939 & Stevng & 2 \\
\hline Cardff & 674 & & $\mathrm{n} / \mathrm{a}$ \\
\hline Covnt & 333 & & $\mathrm{n} / \mathrm{a}$ \\
\hline L Barts & 652 & & $\mathrm{n} / \mathrm{a}$ \\
\hline L Guys & 1,076 & Kent & 3 \\
\hline L Rfree & 476 & & $\mathrm{n} / \mathrm{a}$ \\
\hline L St.G & 367 & Carsh & 14 \\
\hline L West & 1,047 & & $\mathrm{n} / \mathrm{a}$ \\
\hline Leeds & 910 & & $\mathrm{n} / \mathrm{a}$ \\
\hline Leic & 479 & & $\mathrm{n} / \mathrm{a}$ \\
\hline Liv RI & 541 & Prestn & 1 \\
\hline M RI & 652 & Salford & 23 \\
\hline Newc & 735 & & $\mathrm{n} / \mathrm{a}$ \\
\hline Nottm & 334 & & $\mathrm{n} / \mathrm{a}$ \\
\hline Oxford & 953 & & $\mathrm{n} / \mathrm{a}$ \\
\hline Plymth & 388 & & $\mathrm{n} / \mathrm{a}$ \\
\hline Ports & 412 & & $\mathrm{n} / \mathrm{a}$ \\
\hline Sheff & 363 & & $\mathrm{n} / \mathrm{a}$ \\
\hline Total & 13,124 & & 61 \\
\hline
\end{tabular}

${ }^{\star}$ Only transplant centres in England, N Ireland and Wales included 


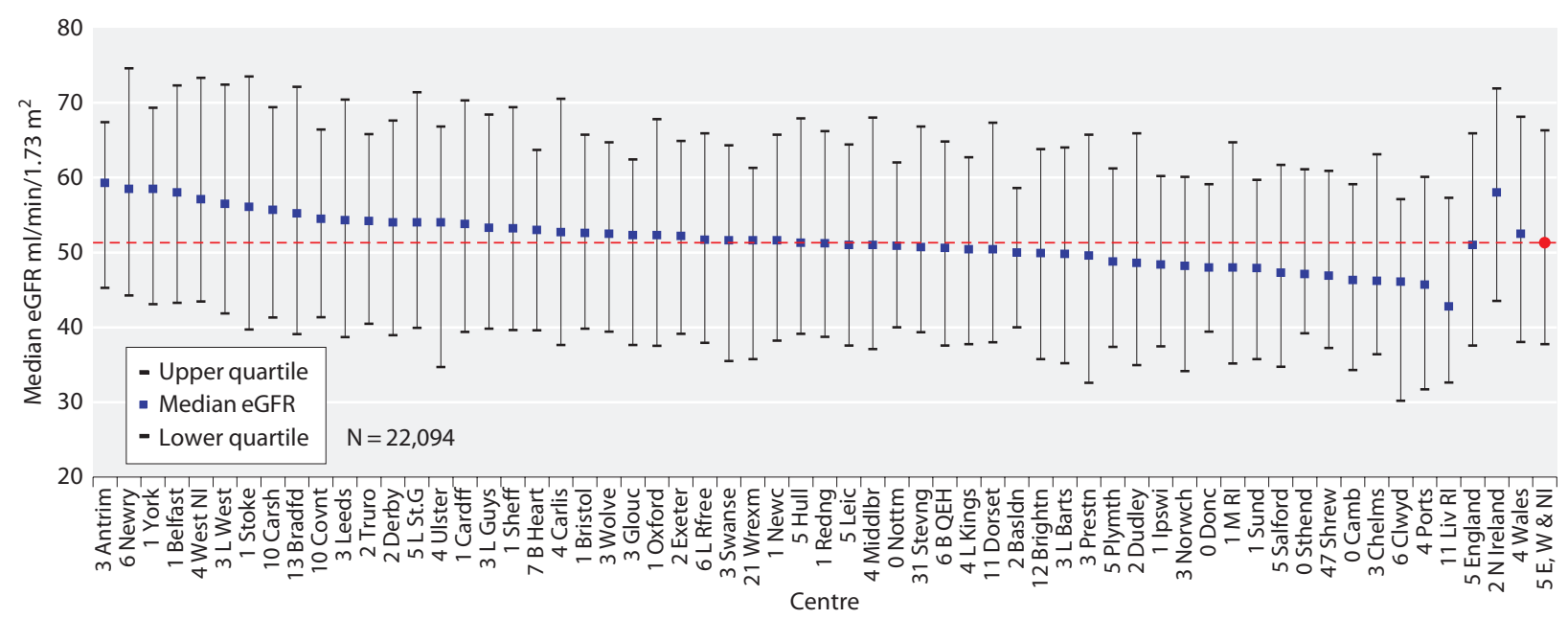

Fig. 3.2. Median eGFR in prevalent transplant patients by centre on 31/12/2011

only have a modest predictive performance in the transplant population [3]. Median eGFR in each centre and percentage of patients with eGFR $<30 \mathrm{ml} / \mathrm{min} / 1.73 \mathrm{~m}^{2}$ are shown in figures 3.2 and 3.3. The median eGFR was $51.3 \mathrm{ml} / \mathrm{min} / 1.73 \mathrm{~m}^{2}$, with $13.6 \%$ of prevalent transplant recipients having an eGFR $<30 \mathrm{ml} / \mathrm{min} / 1.73 \mathrm{~m}^{2}$. Table 3.11 summarises the proportion of transplant patients with an eGFR $<30 \mathrm{ml} / \mathrm{min} / 1.73 \mathrm{~m}^{2}$ by centre. Whilst local repatriation policies on timing of transfer of care for patients with failing transplants from transplant centres to referring centres might explain some of the differences, it is notable that both transplanting and nontransplanting centres feature at both ends of the scale. The accuracy of the 4-variable MDRD equation in estimating GFR $\geqslant 60 \mathrm{ml} / \mathrm{min} / 1.73 \mathrm{~m}^{2}$ is questionable [4], therefore a figure describing this is not included in this chapter.

Figure 3.4 shows the percentage of prevalent patients by centre with eGFR $<30 \mathrm{ml} / \mathrm{min} / 1.73 \mathrm{~m}^{2}$ as a funnel plot, enabling a more reliable comparison of outcomes between centres across the UK. The solid lines show the 2 standard deviation limits (95\%) and the dotted lines the limits for 3 standard deviations (99.9\%). With 58 centres included and a normal distribution, $2-3$ centres would be expected to fall between the $95 \%-99 \%$ CI ( 1 in 20) and no centres should fall outside the $99.9 \%$ limits.

There continued to be variation between centres; these data show over-dispersion with 15 centres falling outside the $95 \%$ CI of which eight centres were outside the $99.9 \%$ CI. Five centres (Bristol, Belfast, Newry, London West,

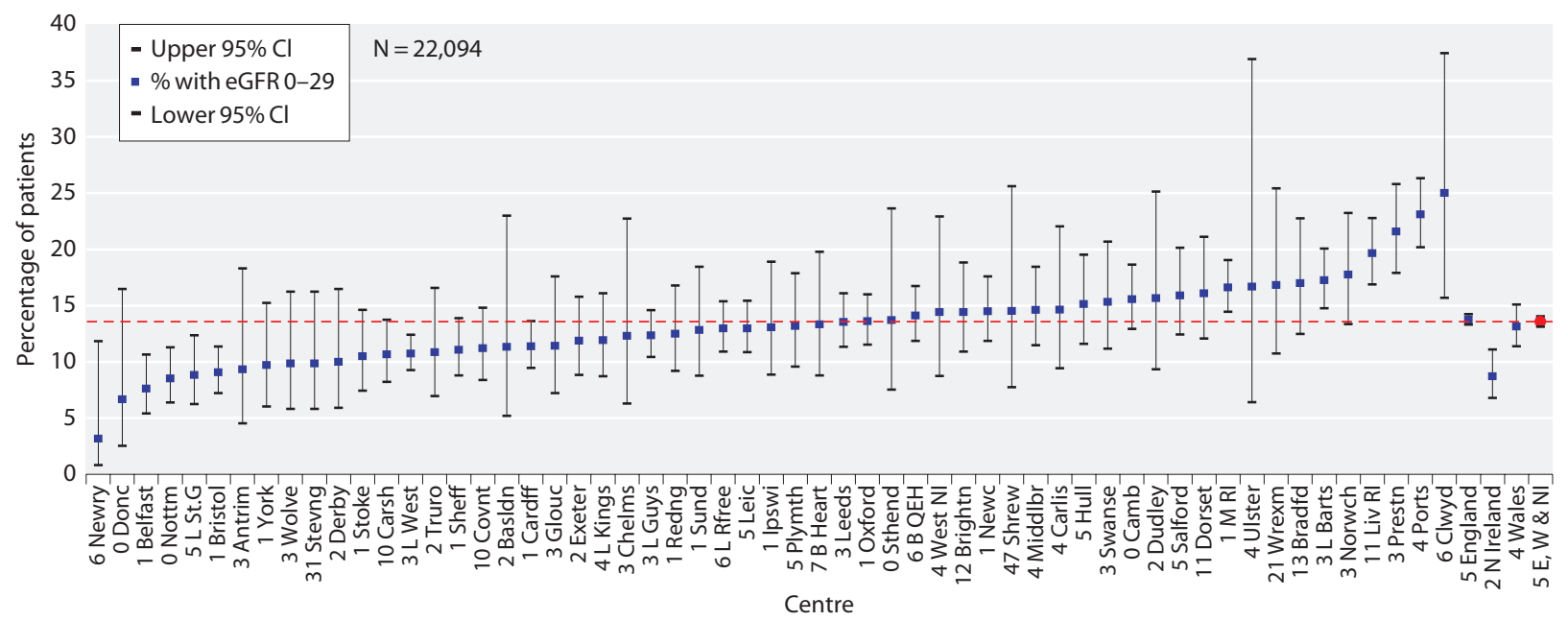

Fig. 3.3. Percentage of prevalent transplant patients by centre on $31 / 12 / 2011$ with eGFR $<30 \mathrm{ml} / \mathrm{min} / 1.73 \mathrm{~m}{ }^{2}$ 
Table 3.11. Proportion of prevalent transplant patients with eGFR $<30 \mathrm{ml} / \mathrm{min} / 1.73 \mathrm{~m}^{2}$ on $31 / 12 / 2011$

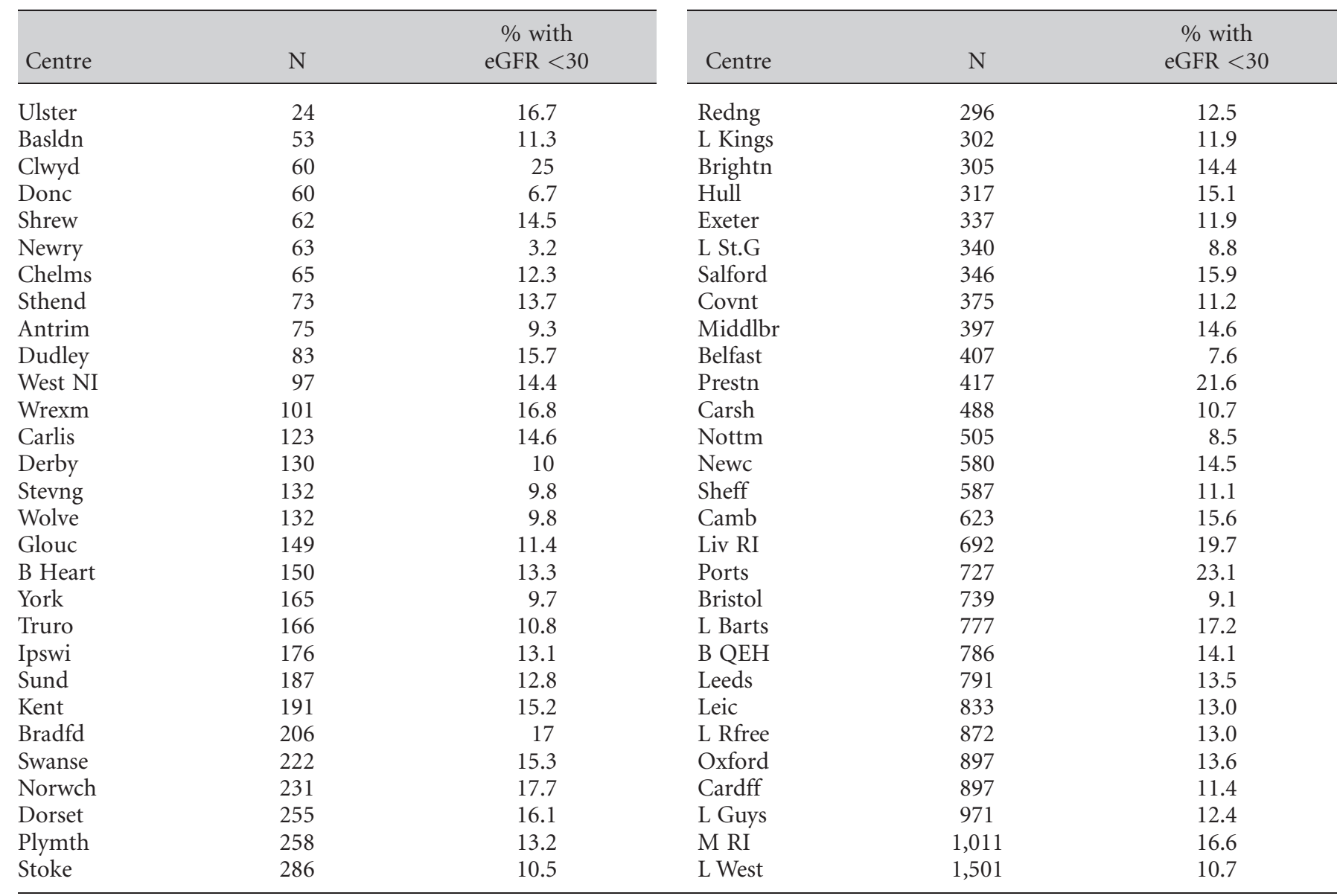

Nottingham) fell outside the lower $99.9 \%$ CI suggesting a lower than expected proportion of patients with eGFR $<30 \mathrm{ml} / \mathrm{min} / 1.73 \mathrm{~m}^{2}$. Liverpool RI, Portsmouth and Preston fell outside the upper $99.9 \%$ CI suggesting a higher than expected proportion of patients with eGFR $<30 \mathrm{ml} / \mathrm{min} / 1.73 \mathrm{~m}^{2}$.

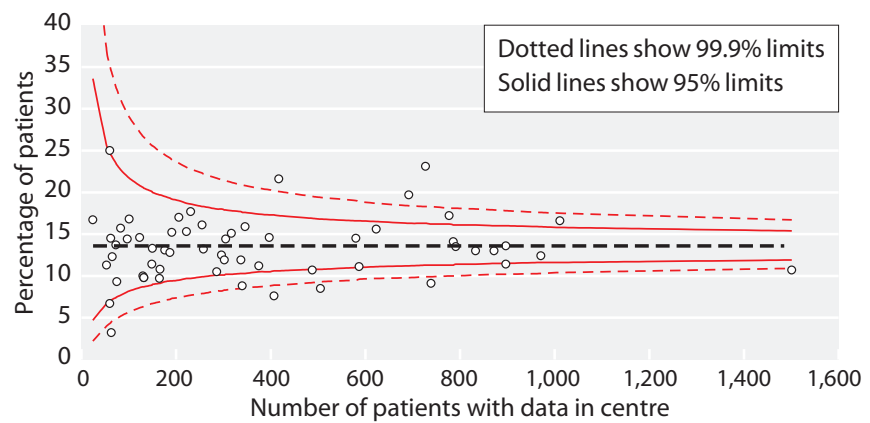

Fig. 3.4. Funnel plot of percentage of prevalent transplant patients with eGFR $<30 \mathrm{ml} / \mathrm{min} / 1.73 \mathrm{~m}^{2}$ by centre size on $31 / 12 / 2011$
eGFR in patients one year after transplantation

Graft function at one year post-transplantation may predict subsequent long-term graft outcome [5]. Figures $3.5 \mathrm{a}, 3.5 \mathrm{~b}$, and $3.5 \mathrm{c}$ show the median one year posttransplant eGFR for patients transplanted between 2004-2010, by transplant type. Living kidney donation had the highest median eGFR at one year $(55.9 \mathrm{ml} / \mathrm{min} /$ $1.73 \mathrm{~m}^{2}$ ), followed by donation after brainstem death $\left(51.8 \mathrm{ml} / \mathrm{min} / 1.73 \mathrm{~m}^{2}\right)$ and donation after circulatory death $\left(49.4 \mathrm{ml} / \mathrm{min} / 1.73 \mathrm{~m}^{2}\right)$.

Figures 3.6a, 3.6b and 3.6c show one year posttransplant eGFR by donor type and year of transplantation. An upward trend in eGFR $(\mathrm{p}<0.001)$ over the time period was noticed with both live and donation after brainstem death transplant, but not with donation after circulatory death $(\mathrm{p}=0.1)$.

Haemoglobin in prevalent transplant patients

Transplant patients have previously fallen under the remit of the UK Renal Association Complications of 


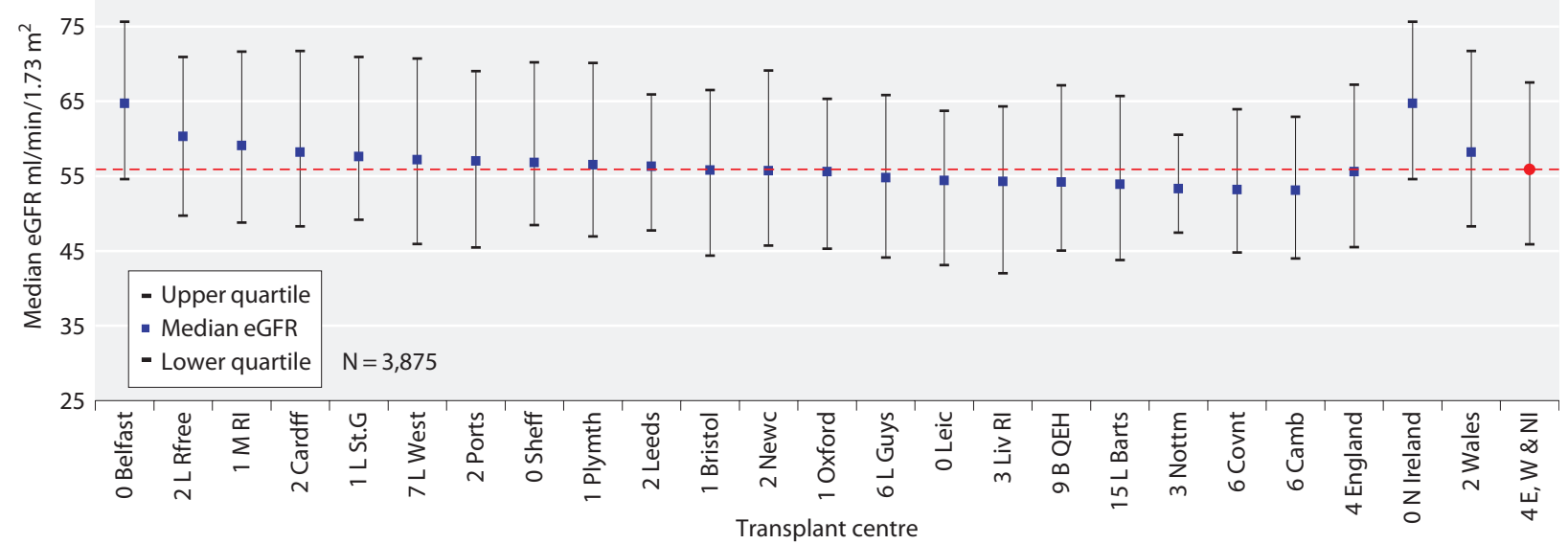

Fig. 3.5a. Median eGFR one year post-live donor transplant by transplant centre 2004-2010

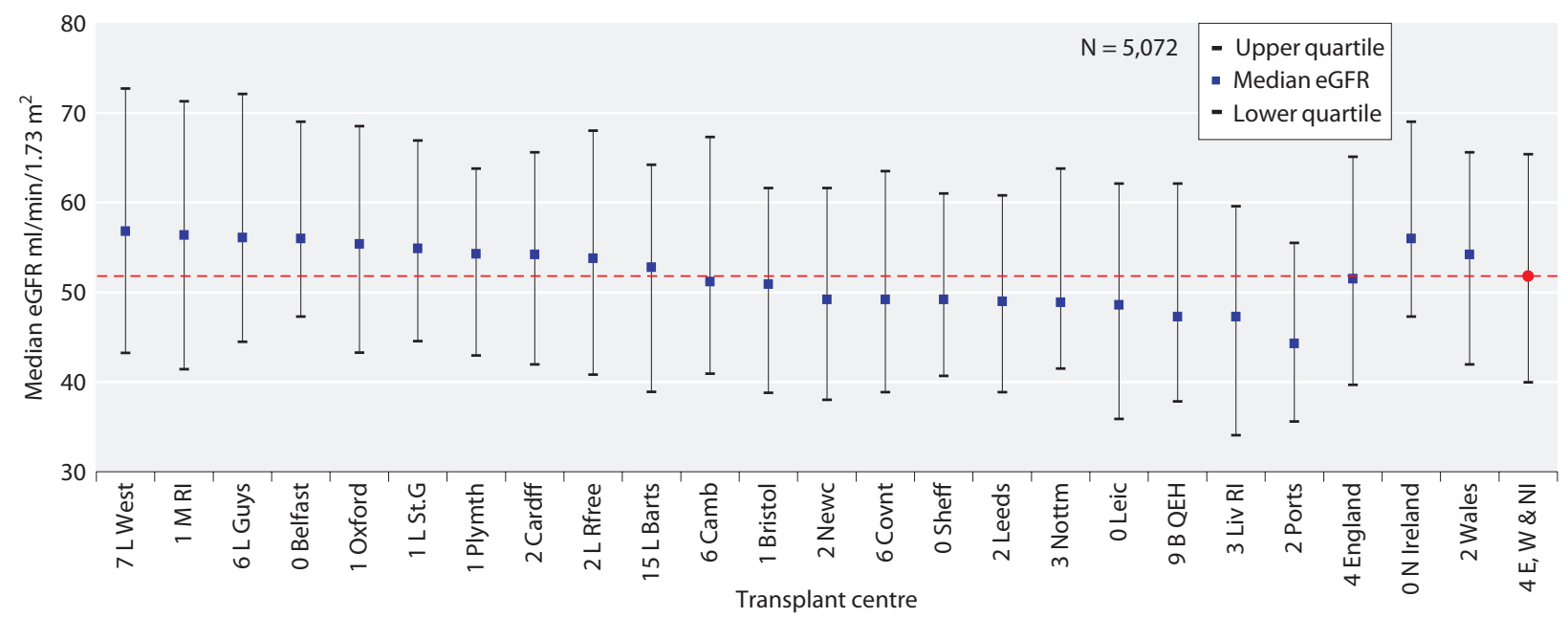

Fig. 3.5b. Median eGFR one year post-brainstem death donor transplant by transplant centre 2004-2010

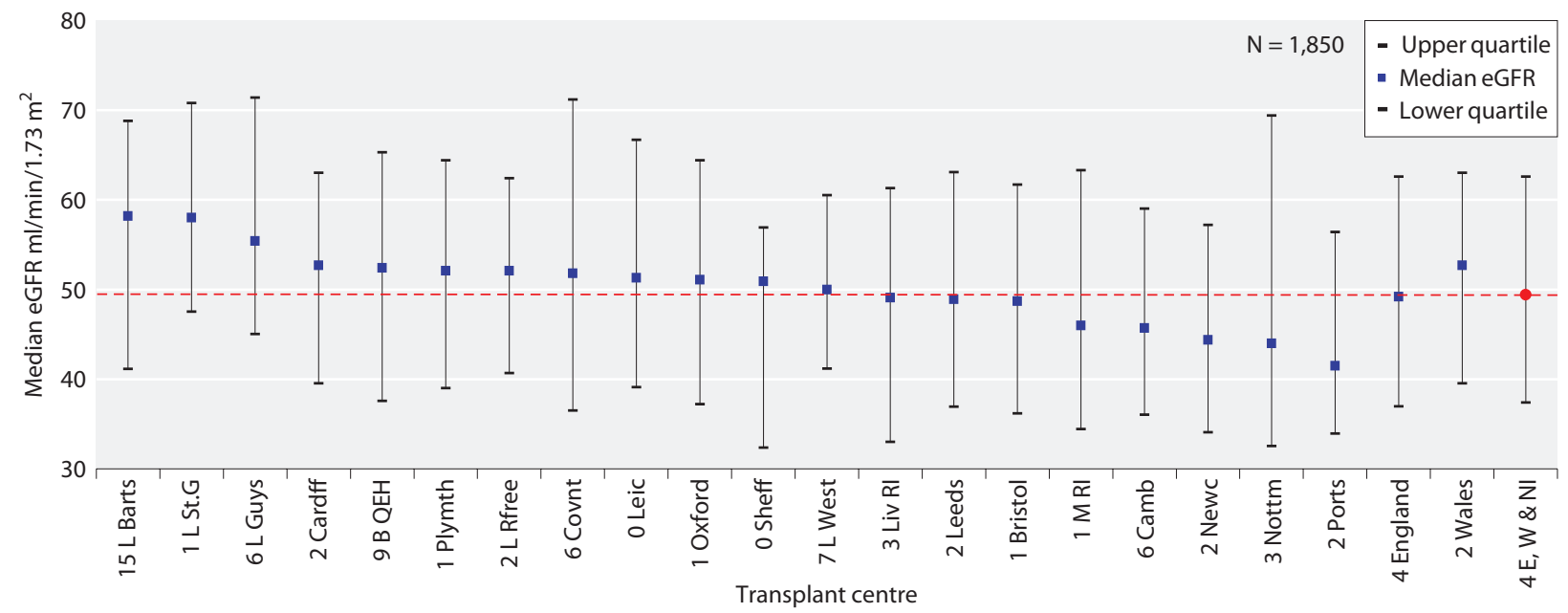

Fig. 3.5c. Median eGFR one year post-circulatory death donor transplant by transplant centre 2004-2010 


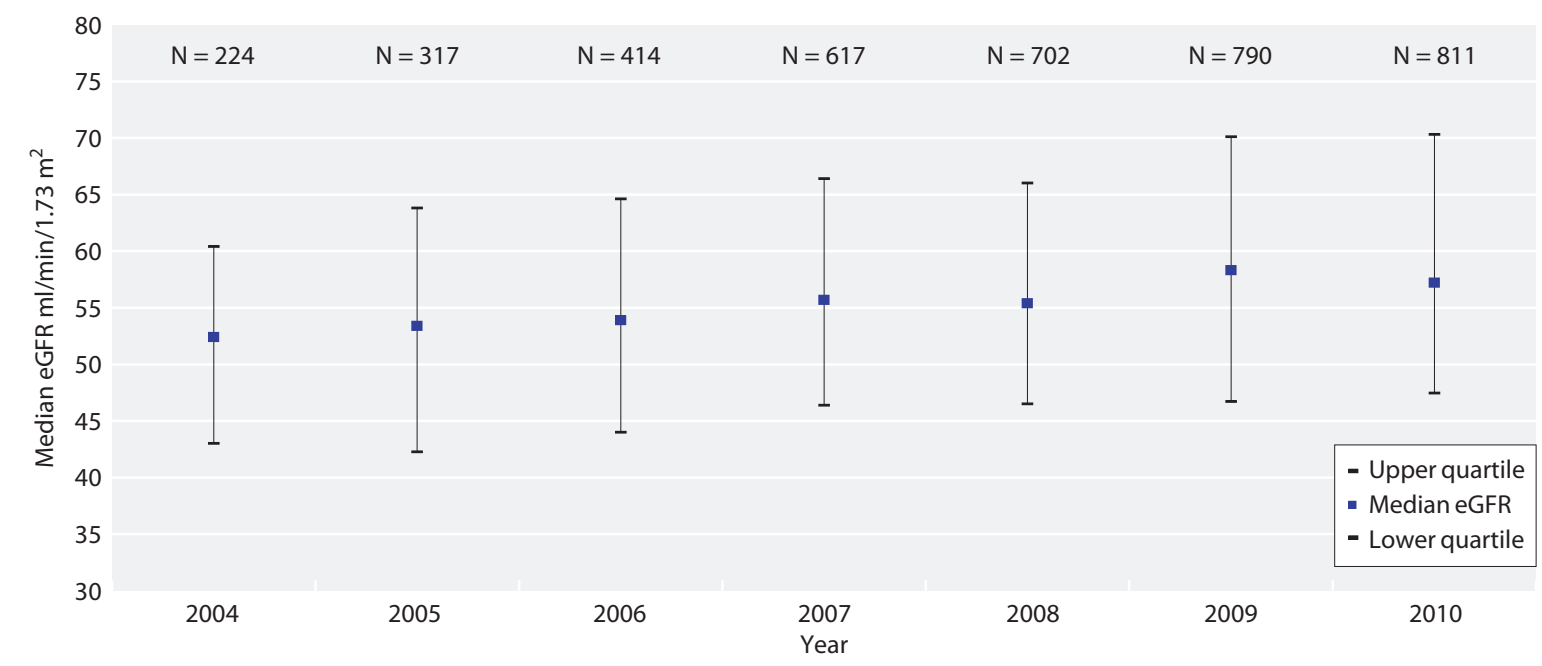

Fig. 3.6a. Median eGFR one year post-live donor transplant by year of transplantation 2004-2010

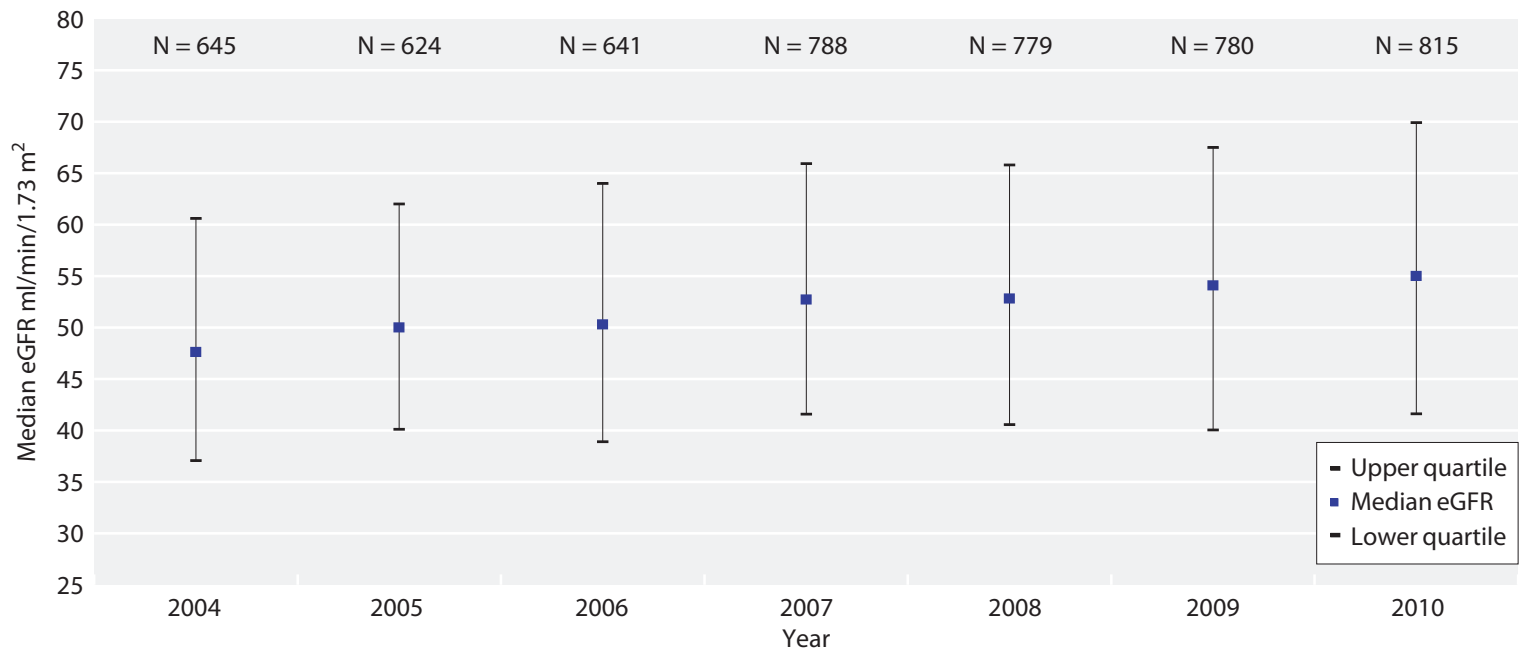

Fig. 3.6b. Median eGFR one year post-brainstem death donor transplant by year of transplantation 2004-2010

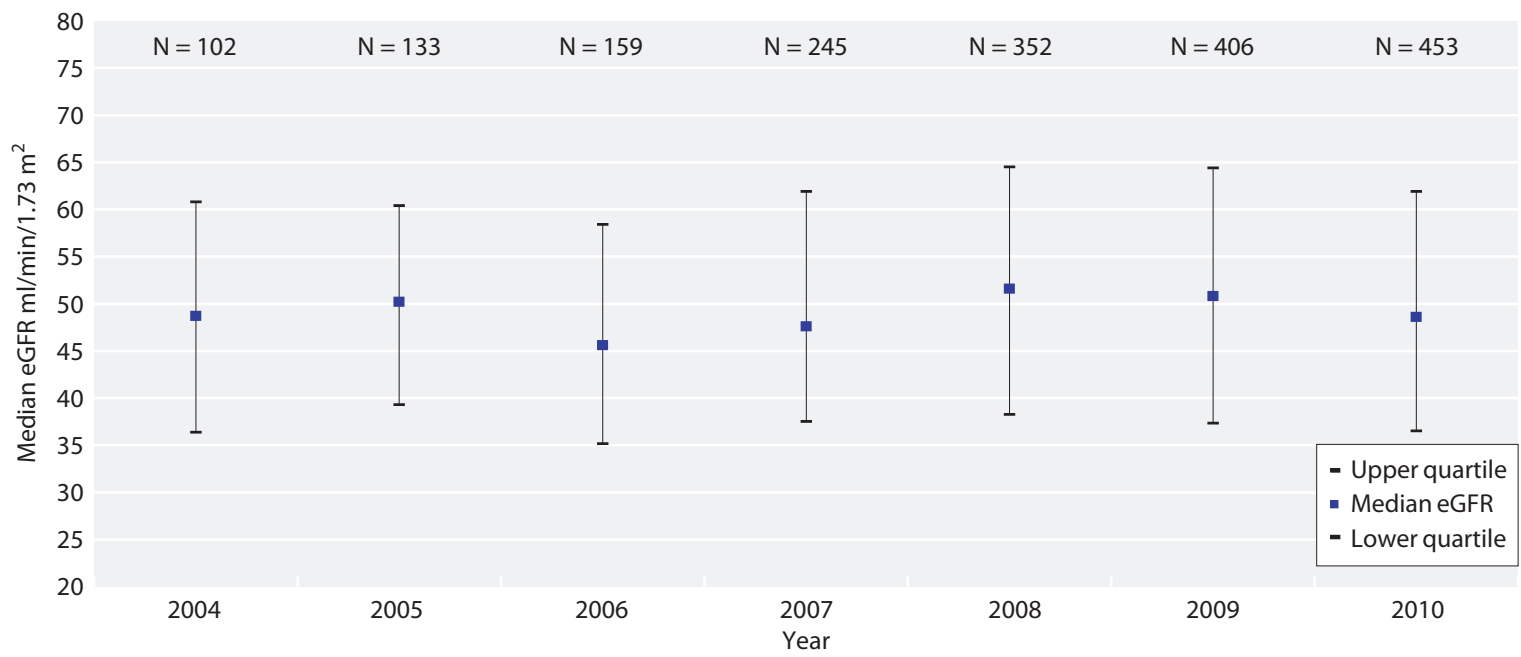

Fig. 3.6c. Median eGFR one year post-circulatory death donor transplant by year of transplantation 2004-2010 


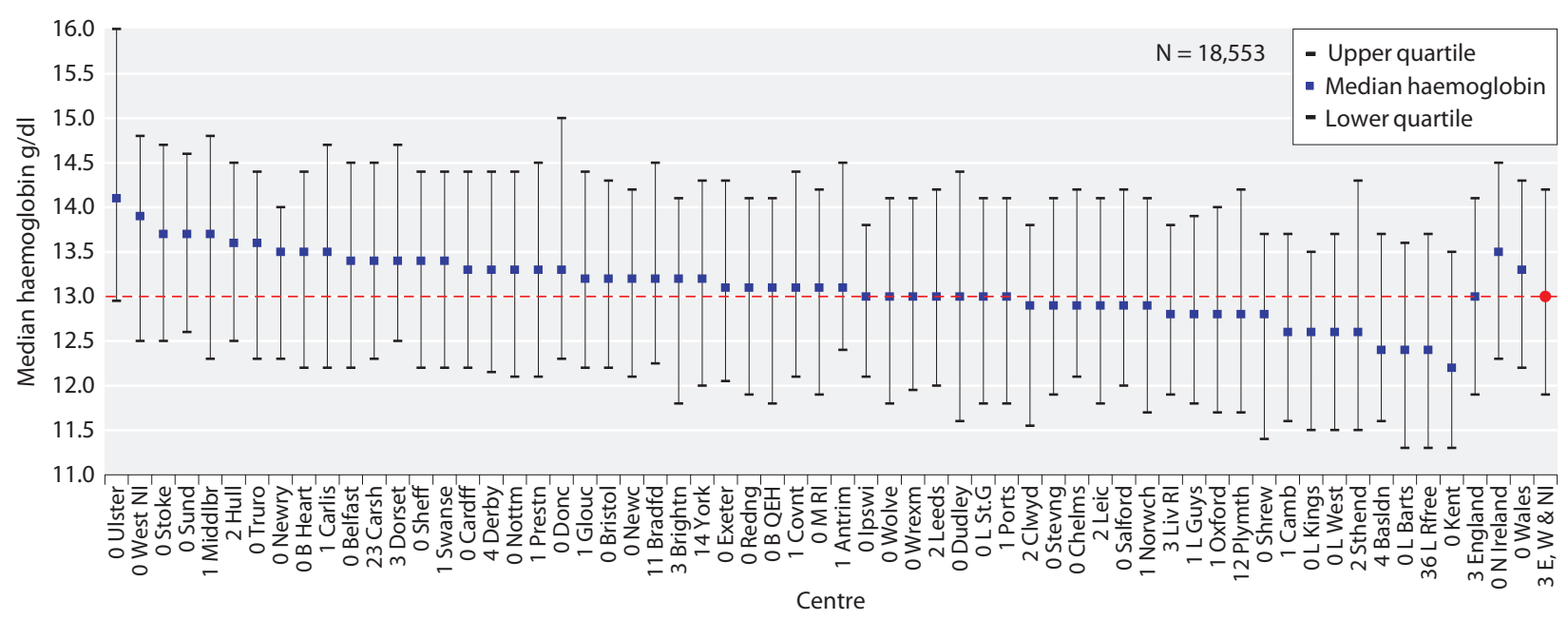

Fig. 3.7a. Median haemoglobin for prevalent transplant patients with eGFR $\geqslant 30 \mathrm{ml} / \mathrm{min} / 1.73 \mathrm{~m}^{2}$ by centre on $31 / 12 / 2011$

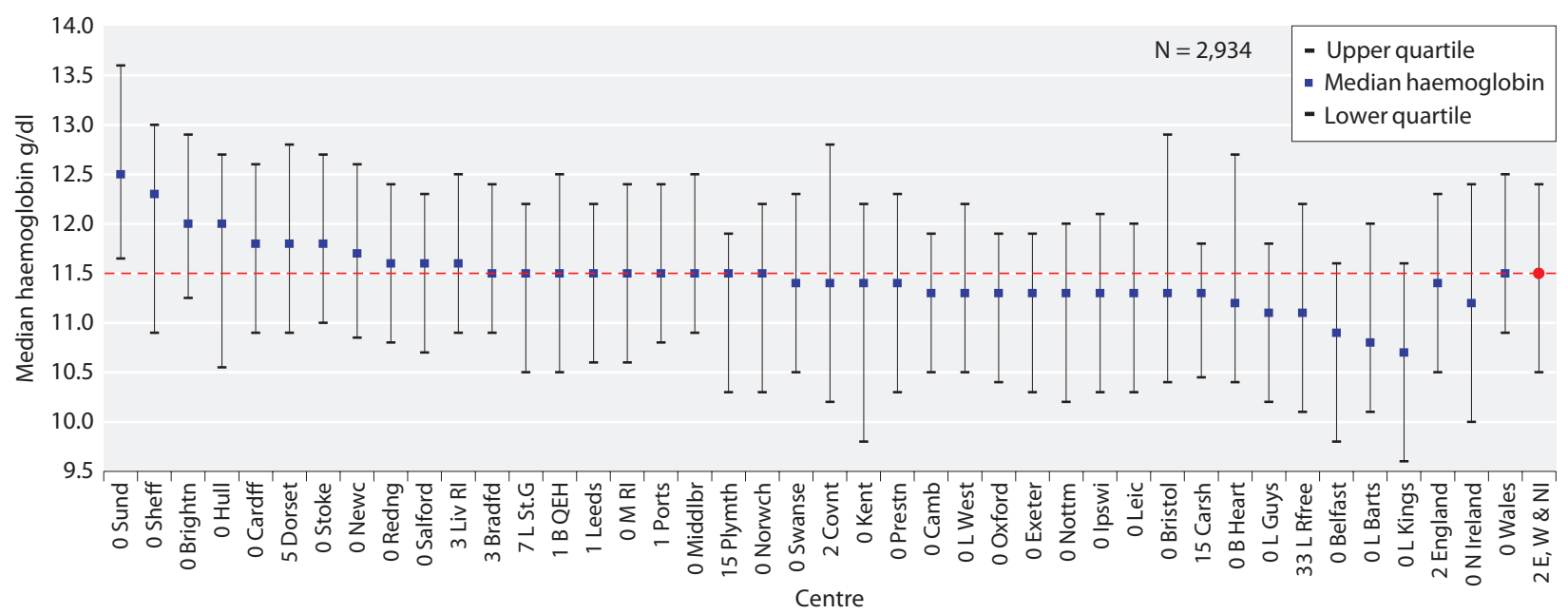

Fig. 3.7b. Median haemoglobin for prevalent transplant patients with eGFR $<30 \mathrm{ml} / \mathrm{min} / 1.73 \mathrm{~m}^{2}$ by centre on $31 / 12 / 2011$

Chronic Kidney Disease (CKD) guidelines. Updated guidelines regarding the management of anaemia in CKD were published by the association in November 2010 [6] which have now been adopted for this report. These guidelines recommend achieving a population distribution centred on a mean of $11 \mathrm{~g} / \mathrm{dl}$ with a range of 10-12 g/dl [7]. However, many transplant patients with good transplant function will have haemoglobin concentrations $>12 \mathrm{~g} / \mathrm{dl}$ without the use of erythopoiesis stimulating agents, and so it is inappropriate to audit performance using the higher limit.

A number of factors including comorbidity, immunosuppressive medication, graft function, ACE inhibitor use, erythropoietin (EPO) use, intravenous or oral iron use, as well as centre practices and protocols for management of anaemia, affect haemoglobin concentrations in transplant patients. Most of these data are not collected by the UKRR and therefore caution must be used when interpreting analyses of haemoglobin attainment. Figures $3.7 \mathrm{a}$ and $3.7 \mathrm{~b}$ report centre results stratified according to graft function as estimated by eGFR. The percentage of prevalent transplant patients achieving $\mathrm{Hb} \geqslant 10.0 \mathrm{~g} / \mathrm{dl}$ in each centre, stratified by eGFR, is displayed in figures $3.8 \mathrm{a}$ and $3.8 \mathrm{~b}$.

Figure 3.9 describes the percentage of prevalent patients by centre with haemoglobin $<10.0 \mathrm{~g} / \mathrm{dl}$ as a funnel plot enabling more reliable comparison of outcomes between centres across the UK. With 58 centres included and a normal distribution, $2-3$ centres would be expected to fall between the 95\%-99.9\% CI ( 1 in 20) and no centres should fall outside the $99.9 \%$ CI purely as a chance event. 


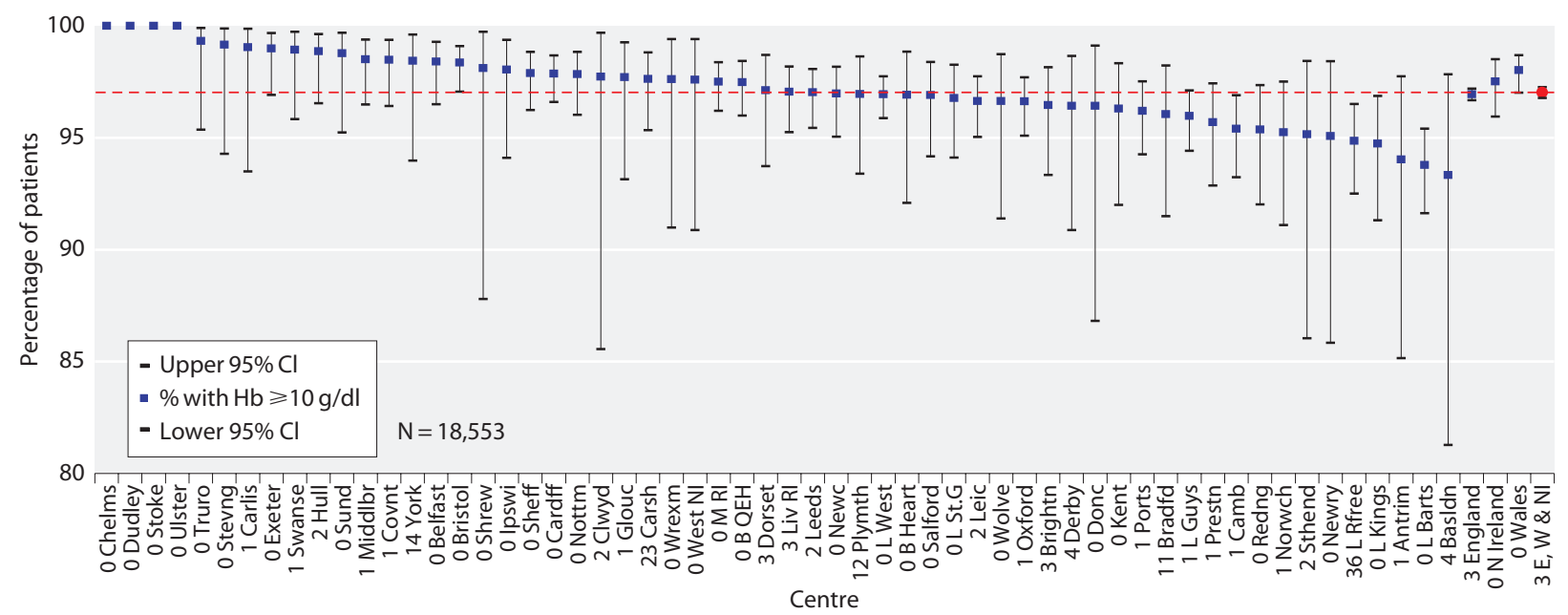

Fig. 3.8a. Percentage of prevalent transplant patients with eGFR $\geqslant 30 \mathrm{ml} / \mathrm{min} / 1.73 \mathrm{~m}^{2}$ achieving haemoglobin $\geqslant 10.0 \mathrm{~g} / \mathrm{dl}$ by centre on $31 / 12 / 2011$

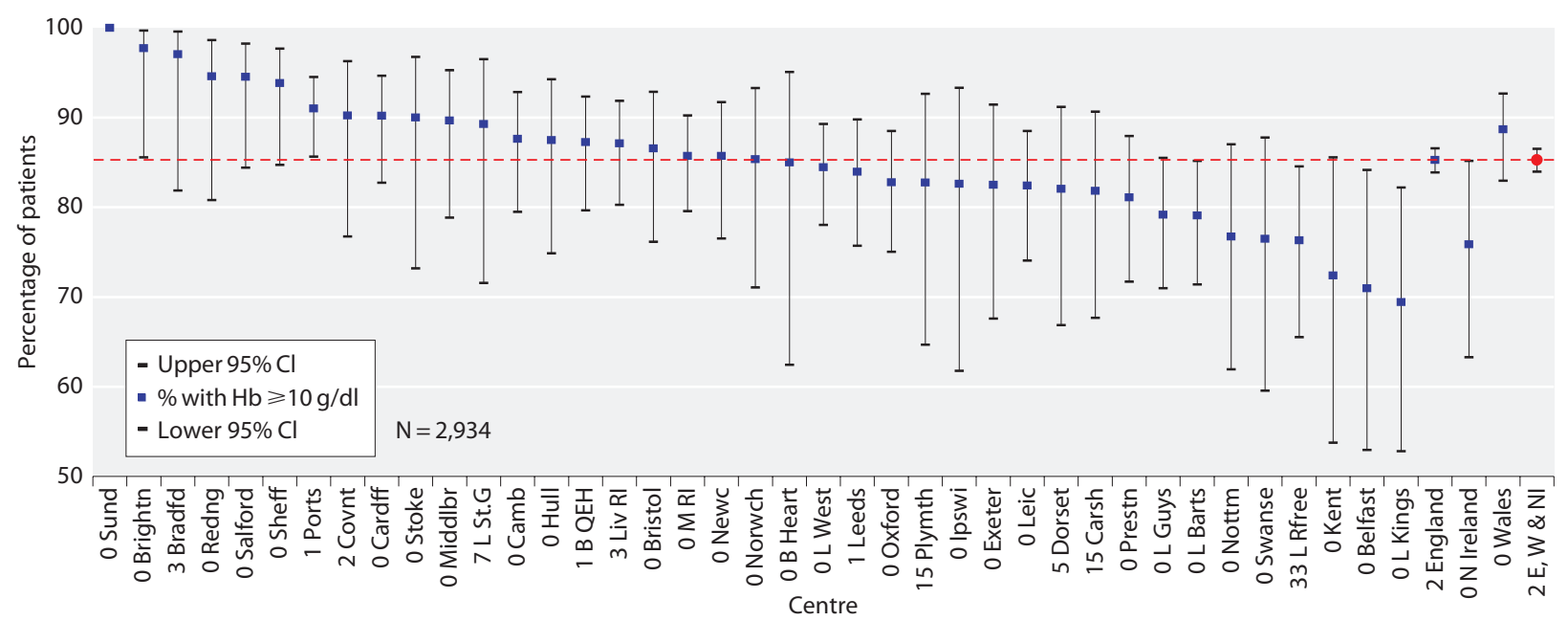

Fig. 3.8b. Percentage of prevalent transplant patients with eGFR $<30 \mathrm{ml} / \mathrm{min} / 1.73 \mathrm{~m}^{2}$ achieving haemoglobin $\geqslant 10.0 \mathrm{~g} / \mathrm{dl}$ by centre on $31 / 12 / 2011$

One centre (London Barts) fell outside the upper 99.9\% CI and three further centres (London Kings, London Royal Free and Preston) fell outside the upper 95\% CI indicating a higher than predicted proportion of transplant patients not achieving the haemoglobin target. Three centres fell outside the lower $99.9 \%$ CI, indicating they performed better than expected with fewer than predicted patients having a haemoglobin $<10.0 \mathrm{~g} / \mathrm{dl}$.

Blood pressure in prevalent transplant patients

In the absence of controlled trial data, the opinionbased recommendation of the UK Renal Association (RA) published in the 2010 guideline for the care of

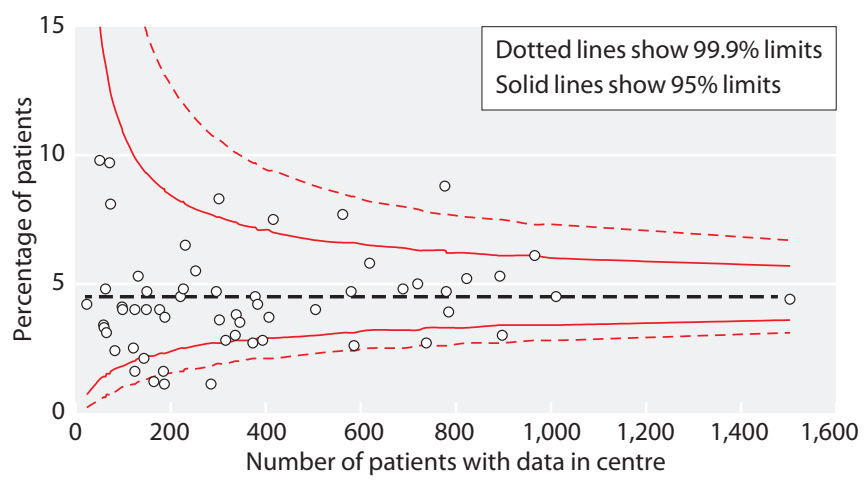

Fig. 3.9. Funnel plot of percentage of prevalent transplant patients with haemoglobin $<10.0 \mathrm{~g} /$ dl by centre size on $31 / 12 / 2011$ 


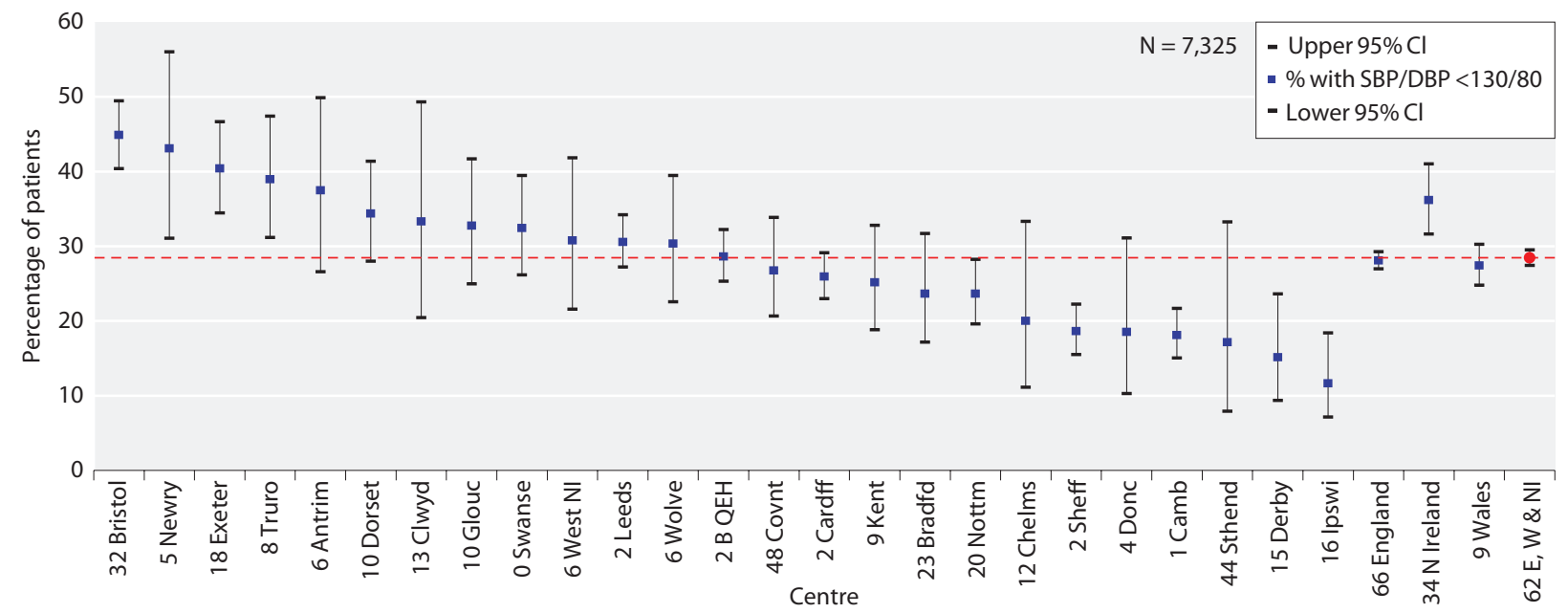

Fig. 3.10a. Percentage of prevalent transplant patients with eGFR $\geqslant 30 \mathrm{ml} / \mathrm{min} / 1.73 \mathrm{~m}^{2}$ achieving blood pressure of $<130 / 80 \mathrm{mmHg}$ by centre on $31 / 12 / 2011$

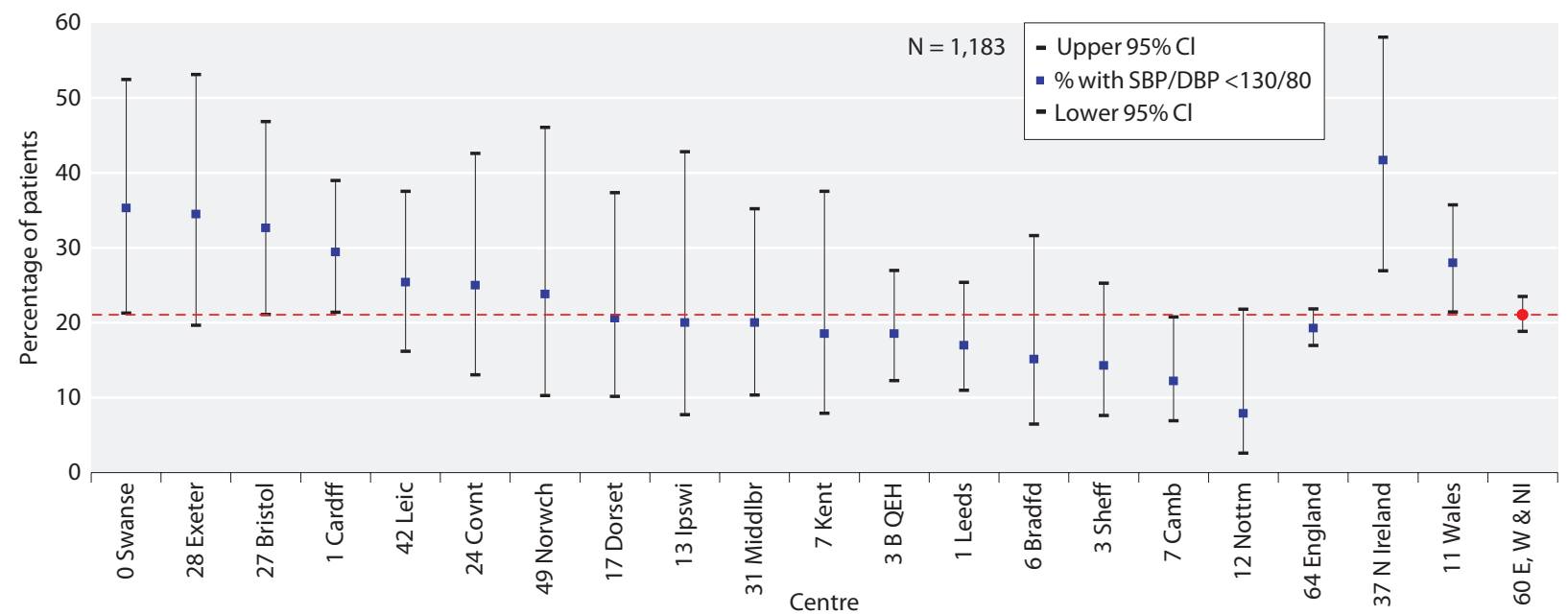

Fig. 3.10b. Percentage of prevalent transplant patients with eGFR $<30 \mathrm{ml} / \mathrm{min} / 1.73 \mathrm{~m}^{2}$ achieving blood pressure of $<130 / 80 \mathrm{mmHg}$ by centre on $31 / 12 / 2011$

the kidney transplant recipients is that 'Blood pressure should be $<130 / 80 \mathrm{mmHg}$ (or $<125 / 75 \mathrm{mmHg}$ if proteinuria)' [8]. This blood pressure target is the same as that used in previous annual reports [9].

As indicated in table 3.9a, completeness for blood pressure data returns was variable and only centres with $>50 \%$ data returns were included for consideration. Despite this restriction, caution needs to be exercised in interpretation of these results because of the volume of missing data and potential bias, (e.g. a centre may be more likely to record and report blood pressure data electronically in patients with poor BP control). Figures $3.10 \mathrm{a}$ and $3.10 \mathrm{~b}$ show the percentage of patients with a blood pressure of $<130 / 80 \mathrm{mmHg}$, by eGFR. The percentage of patients with $\mathrm{BP}<130 / 80$ (systolic BP
$<130$ and diastolic BP $<80 \mathrm{mmHg}$ ) was higher $(28.5 \%$ vs. $21.1 \%$ ) in those with better renal function $\left(\mathrm{eGFR} \geqslant 30 \mathrm{ml} / \mathrm{min} / 1.73 \mathrm{~m}^{2}\right)$.

\section{Analysis of prevalent patients by CKD stage}

\section{Introduction}

Approximately $2.2 \%$ of prevalent transplant patients returned to dialysis in 2011, a similar percentage to that seen over the last few years. Amongst patients with native chronic kidney disease, late presentation is associated with poor outcomes, largely attributable to lack of specialist management of anaemia, acidosis, 
hyperphosphataemia and to inadequate advance preparation for dialysis. Transplant recipients on the other hand, are almost always followed up regularly in specialist transplant or renal clinics and it would be reasonable to expect patients with failing grafts to receive appropriate care and therefore have many of their modifiable risk factors addressed before complete graft failure and return to dialysis.

\section{Methods}

The transplant cohort consisted of prevalent transplant recipients as on 31st December $2011(\mathrm{~N}=22,109)$ and were classified according to the KDIGO staging criteria with the suffix of ' $T$ ' to represent their transplant status. Patients with missing ethnicity information were classified as White for the purpose of calculating eGFR. Prevalent dialysis patients, except those who commenced dialysis in 2011, comprised the comparison dialysis cohort $(\mathrm{N}=19,150)$ including 2,241 peritoneal dialysis patients. Only patients on peritoneal dialysis were considered when examining differences in serum phosphate between transplant recipients and dialysis patients. For both the transplant and dialysis cohorts, the analysis used the most recent available value from the last two quarters of the 2011 laboratory data.

\section{Results and discussion}

Table 3.12 shows that $13.6 \%$ of the prevalent transplant population (3,005 patients), had moderate to advanced renal impairment of eGFR $<30 \mathrm{ml} / \mathrm{min} /$ $1.73 \mathrm{~m}^{2}$. The table also demonstrates that patients with failing grafts achieved UK Renal Association standards for some key biochemical and clinical outcome variables less often than dialysis patients. This substantial group of patients represents a considerable challenge, as resources need to be channelled to improve key outcome variables and achieve a safe and timely modality switch to another form of renal replacement therapy.

Table 3.12. Analysis by CKD stage for prevalent transplant patients compared with prevalent dialysis patients on $31 / 12 / 2011$

\begin{tabular}{|c|c|c|c|c|c|}
\hline & $\begin{array}{l}\text { Stage } 1-2 \mathrm{~T} \\
\quad(\geqslant 60)\end{array}$ & $\begin{array}{l}\text { Stage 3T } \\
(30-59)\end{array}$ & $\begin{array}{l}\text { Stage 4T } \\
(15-29)\end{array}$ & $\begin{array}{l}\text { Stage } 5 \mathrm{~T} \\
(<15)\end{array}$ & Stage 5D \\
\hline Number of patients & 7,603 & 11,501 & 2,635 & 370 & 19,150 \\
\hline $\begin{array}{l}\text { eGFR } \mathbf{~ m l} / \mathrm{min} / 1.73 \mathrm{~m}^{2} \text { a } \\
\text { mean } \pm S D \\
\text { median }\end{array}$ & $\begin{array}{c}76.8 \pm 15.2 \\
72.7\end{array}$ & $\begin{array}{c}45.6 \pm 8.4 \\
45.8\end{array}$ & $\begin{array}{c}23.9 \pm 4.2 \\
24.5\end{array}$ & $\begin{array}{c}11.9 \pm 2.3 \\
12.2\end{array}$ & \\
\hline $\begin{array}{l}\text { Diastolic BP } \mathbf{m m H g} \\
\text { mean } \pm \text { SD } \\
\% \geqslant 80\end{array}$ & $\begin{array}{c}77.8 \pm 10.0 \\
45.8\end{array}$ & $\begin{array}{c}78.0 \pm 10.1 \\
46.9\end{array}$ & $\begin{array}{c}78.0 \pm 11.0 \\
48.2\end{array}$ & $\begin{array}{c}78.7 \pm 11.3 \\
51.0\end{array}$ & $\begin{array}{c}68.4 \pm 14.5 \\
21.7\end{array}$ \\
\hline $\begin{array}{l}\text { Haemoglobin } \mathbf{g} / \mathbf{d l} \\
\text { mean } \pm \text { SD } \\
\%<10.0\end{array}$ & $\begin{array}{c}13.6 \pm 1.6 \\
1.6\end{array}$ & $\begin{array}{c}12.7 \pm 1.6 \\
3.9\end{array}$ & $\begin{array}{c}11.6 \pm 1.5 \\
12.0\end{array}$ & $\begin{array}{c}10.6 \pm 1.6 \\
34.0\end{array}$ & $\begin{array}{c}11.2 \pm 1.4 \\
16.7\end{array}$ \\
\hline $\begin{array}{l}\text { Phosphate } \mathbf{~ m m o l} / \mathbf{L}^{\mathbf{b}} \\
\text { mean } \pm \mathrm{SD} \\
\% \geqslant 1.8\end{array}$ & $\begin{array}{c}0.9 \pm 0.2 \\
0.0\end{array}$ & $\begin{array}{c}1.0 \pm 0.2 \\
0.1\end{array}$ & $\begin{array}{c}1.2 \pm 0.3 \\
1.6\end{array}$ & $\begin{array}{c}1.5 \pm 0.4 \\
19.8\end{array}$ & $\begin{array}{c}1.6 \pm 0.4 \\
27.0\end{array}$ \\
\hline $\begin{array}{l}\text { Corrected calcium } \mathbf{m m o l} / \mathrm{L} \\
\text { mean } \pm \mathrm{SD} \\
\%>2.6 \\
\%<2.2\end{array}$ & $\begin{array}{l}2.4 \pm 0.2 \\
8.5 \\
8.2\end{array}$ & $\begin{array}{l}2.4 \pm 0.2 \\
8.5 \\
8.3\end{array}$ & $\begin{array}{c}2.4 \pm 0.2 \\
5.4 \\
14.6\end{array}$ & $\begin{array}{c}2.3 \pm 0.2 \\
7.1 \\
21.8\end{array}$ & $\begin{array}{c}2.3 \pm 0.2 \\
6.3 \\
18.9\end{array}$ \\
\hline $\begin{array}{l}\text { PTH pmol/L } \\
\text { median } \\
\% \geqslant 32\end{array}$ & $\begin{array}{l}8.7 \\
3.6\end{array}$ & $\begin{array}{l}9.7 \\
5.7\end{array}$ & $\begin{array}{l}15.9 \\
19.7\end{array}$ & $\begin{array}{l}31.3 \\
48.4\end{array}$ & $\begin{array}{l}28.2 \\
44.2\end{array}$ \\
\hline
\end{tabular}

${ }^{\text {a }}$ Prevalent transplant patients with no ethnicity data were classed as White

${ }^{\mathrm{b}}$ Only PD patients included in stage $5 \mathrm{D}, \mathrm{N}=2,241$ 


\section{eGFR slope analysis}

\section{Introduction}

The gradient of deterioration in eGFR (slope) may predict patients likely to have early graft failure. The eGFR slope and its relationship to specific patient characteristics are presented here.

\section{Methods}

Patients from England, Wales or Northern Ireland aged $\geqslant 18$ years receiving a renal transplant between 1st January 2001 and 31st December 2009, were considered for inclusion. A minimum duration of 18 months graft function was required and three or more creatinine measurements from the second year of graft function onwards were used to plot eGFR slope. If a transplant failed but there were at least three creatinine measurements between 18 months post-transplant and graft failure, the patient was included but no creatinine measurements after the quarter preceding the recorded date of transplant failure were analysed.
Slopes were calculated using linear regression, assuming linearity, and the effect of age, ethnicity, gender, diabetes, donor type, year of transplant and current transplant status were analysed. $\mathrm{P}$ values were calculated using the Kruskal-Wallis test. eGFR was calculated using the CKD-EPI equation and results expressed as $\mathrm{ml} / \mathrm{min} / 1.73 \mathrm{~m}^{2} /$ year. The CKD-EPI equation was used in preference to the MDRD formula as it is thought to have a greater degree of accuracy at higher levels of eGFR [11].

\section{Results and discussion}

The study cohort consisted of 11,664 patients. The median GFR slope was $-0.49 \mathrm{ml} / \mathrm{min} / 1.73 \mathrm{~m}^{2} /$ year (table 3.13). The gradient was steeper for Black recipients $\left(-1.17 \mathrm{ml} / \mathrm{min} / 1.73 \mathrm{~m}^{2} /\right.$ year$)$, in keeping with previously published data suggesting poorer outcomes for this group $[12,13]$. eGFR slope was steeper in recipients of deceased donor kidneys $\left(-0.51 \mathrm{ml} / \mathrm{min} / 1.73 \mathrm{~m}^{2} /\right.$ year $)$ compared to patients who received organs from live donors $\left(-0.47 \mathrm{ml} / \mathrm{min} / 1.73 \mathrm{~m}^{2} /\right.$ year $)$ although this $\mathrm{did}$

Table 3.13. Differences in median eGFR slope between prevalent transplant patients

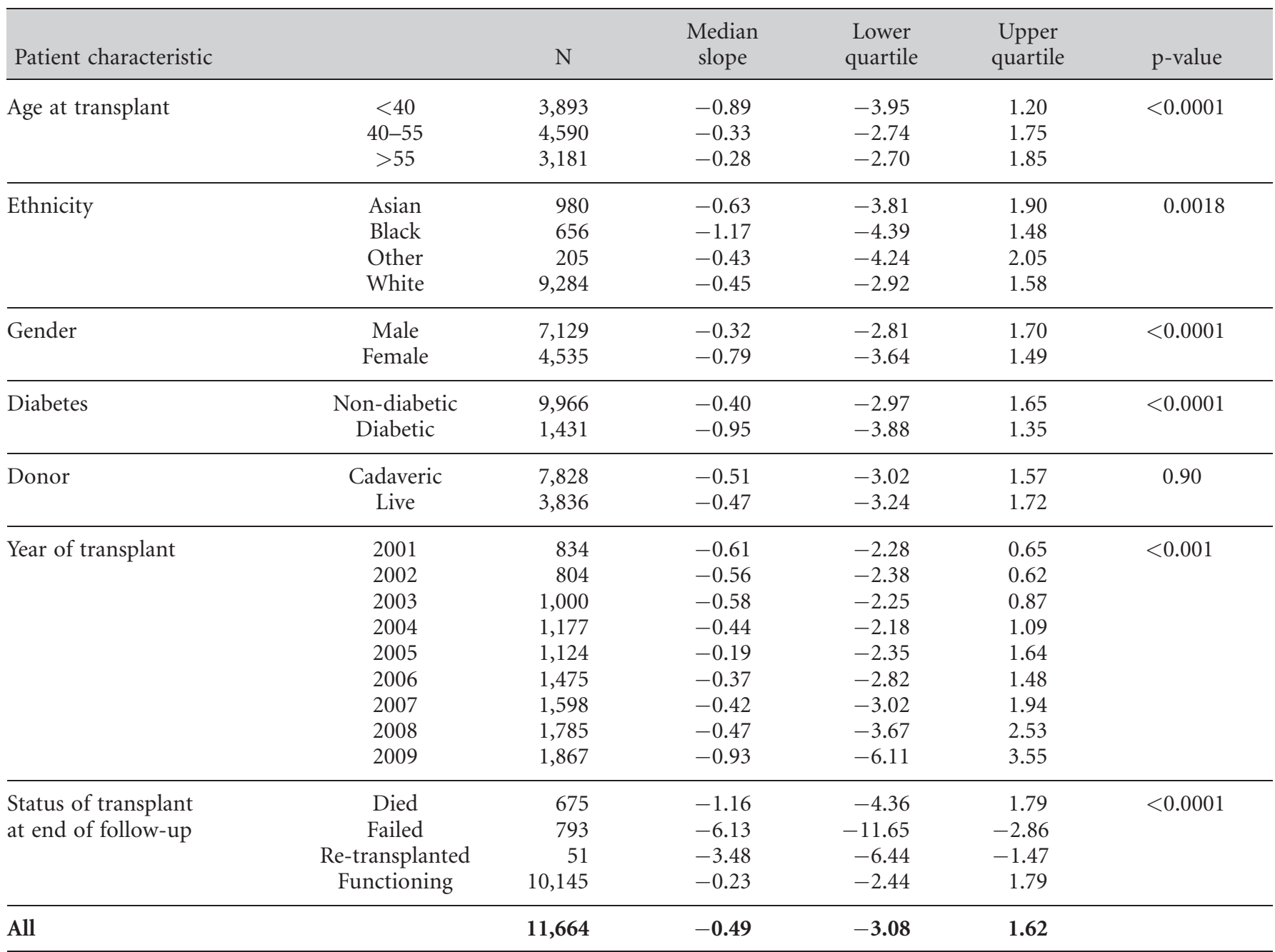


not reach statistical significance. Female patients had a steeper slope $\left(-0.79 \mathrm{ml} / \mathrm{min} / 1.73 \mathrm{~m}^{2} /\right.$ year $)$ than males $\left(-0.32 \mathrm{ml} / \mathrm{min} / 1.73 \mathrm{~m}^{2} /\right.$ year$)$, as did diabetic patients $\left(-0.95 \mathrm{ml} / \mathrm{min} / 1.73 \mathrm{~m}^{2} /\right.$ year$)$ compared to non-diabetic patients $\left(-0.40 \mathrm{ml} / \mathrm{min} / 1.73 \mathrm{~m}^{2} /\right.$ year $)$. The slope was steeper in younger recipients, possibly reflecting increased risk of immunological damage. As might be expected, the steepest slope was in patients where the transplant subsequently failed. This analysis has assumed linearity of progression of fall in GFR and further work is underway to characterise the patterns of progression more precisely.

The findings in this study differ slightly from previous UKRR work exploring eGFR changes in transplant recipients [14]. This identified that male donor to female recipient transplantation, younger recipients, diabetes, white ethnicity, and human leukocyte antigen (HLA) mismatch were associated with faster decline in eGFR. These differences may be explained by patients with eGFR $>60 \mathrm{ml} / \mathrm{min} / 1.73 \mathrm{~m}^{2}$ at one year posttransplantation being excluded and the more complex multivariable model used in the previous work. Udayaraj and colleagues [14] also adjusted for factors such as HLA mismatch and donor age, which were not available for the patients studied in this chapter.

\section{Causes of death in transplant recipients}

\section{Introduction}

Differences in causes of death between dialysis and transplant patients may be expected due to selection for transplantation and use of immunosuppression. Chapter 5 includes a more detailed discussion on causes of death in dialysis patients.

\section{Methods}

The cause of death is sent by renal centres as an ERA-EDTA registry code. These have been grouped into the following categories: cardiac disease, cerebrovascular disease, infection, malignancy, treatment withdrawal, other and uncertain.

This year, individuals with an ERA code 99 (Other identified cause of death) have been removed from category 'Uncertain' (where they were previously coded) to category 'Other' to reflect better coding of the data and bringing the registry in line with the coding methodology adopted in other renal registries. This has substantially reduced the proportion of patient deaths due to 'Uncertain' cause of death with a rise noted in deaths from 'other' causes.

Some centres have high data returns to the UKRR regarding cause of death, whilst others return no information. Provision of this information is not mandatory.

Adult patients aged 18 years and over, from England or Wales, were included in the analyses on cause of death. Previous analyses were limited to data from centres with a high rate of return for cause of death. When this was compared with an analysis of all the cause of death data on the database, the percentages in corresponding ERA-EDTA categories remained unchanged so the latter data were therefore included. Analysis of prevalent patients included all those aged over 18 years and receiving RRT on 31st December 2011.

\section{Results and discussion}

Tables 3.14, 3.15 and figure 3.11 show the differences in the causes of death between prevalent dialysis and transplant patients. Death due to cardiovascular disease was less common in transplanted patients than in dialysis patients, perhaps reflecting the cardiovascular screening undertaken during transplant work-up; transplant recipients are a pre-selected lower risk group of patients. The re-classification of ERA code 99 this year (see methods) has meant that within this cohort the leading cause of death was from 'Other' causes, although similar proportions are seen to have the cause of death attributed to infection and malignancy across all age groups. There has been a reduction over time in the proportion of

Table 3.14. Cause of death by modality in prevalent RRT patients on $1 / 1 / 2011$

\begin{tabular}{|c|c|c|c|c|c|c|}
\hline Cause of death & \multicolumn{2}{|c|}{ All modalities } & \multicolumn{2}{|c|}{ Dialysis } & \multicolumn{2}{|c|}{ Transplant } \\
\hline Cerebrovascular disease & 130 & 5 & 104 & 4 & 26 & 7 \\
\hline Infection & 526 & 19 & 437 & 18 & 89 & 23 \\
\hline Malignancy & 275 & 10 & 193 & 8 & 82 & 21 \\
\hline Uncertain & 115 & 4 & 95 & 4 & 20 & 5 \\
\hline Total & 2,763 & & 2,371 & & 392 & \\
\hline No cause of death data & 1,372 & 33 & 1,138 & 32 & 234 & 37 \\
\hline
\end{tabular}


Table 3.15. Cause of death in prevalent transplant patients on $1 / 1 / 2011$ by age

\begin{tabular}{|c|c|c|c|c|c|c|}
\hline \multirow[b]{2}{*}{ Cause of death } & \multicolumn{2}{|c|}{ All age groups } & \multicolumn{2}{|c|}{$<65$ years } & \multicolumn{2}{|c|}{$\geqslant 65$ years } \\
\hline & $\mathrm{N}$ & $\%$ & $\mathrm{~N}$ & $\%$ & $\mathrm{~N}$ & $\%$ \\
\hline Cardiac disease & 62 & 16 & 34 & 16 & 28 & 16 \\
\hline Cerebrovascular disease & 26 & 7 & 12 & 6 & 14 & 8 \\
\hline Infection & 89 & 23 & 53 & 25 & 36 & 20 \\
\hline Malignancy & 82 & 21 & 42 & 19 & 40 & 23 \\
\hline Treatment withdrawal & 11 & 3 & 6 & 3 & 5 & 3 \\
\hline Other & 102 & 26 & 59 & 27 & 43 & 24 \\
\hline Uncertain & 20 & 5 & 10 & 5 & 10 & 6 \\
\hline Total & 392 & & 216 & & 176 & \\
\hline No cause of death data & 234 & 37 & 117 & 35 & 117 & 40 \\
\hline
\end{tabular}

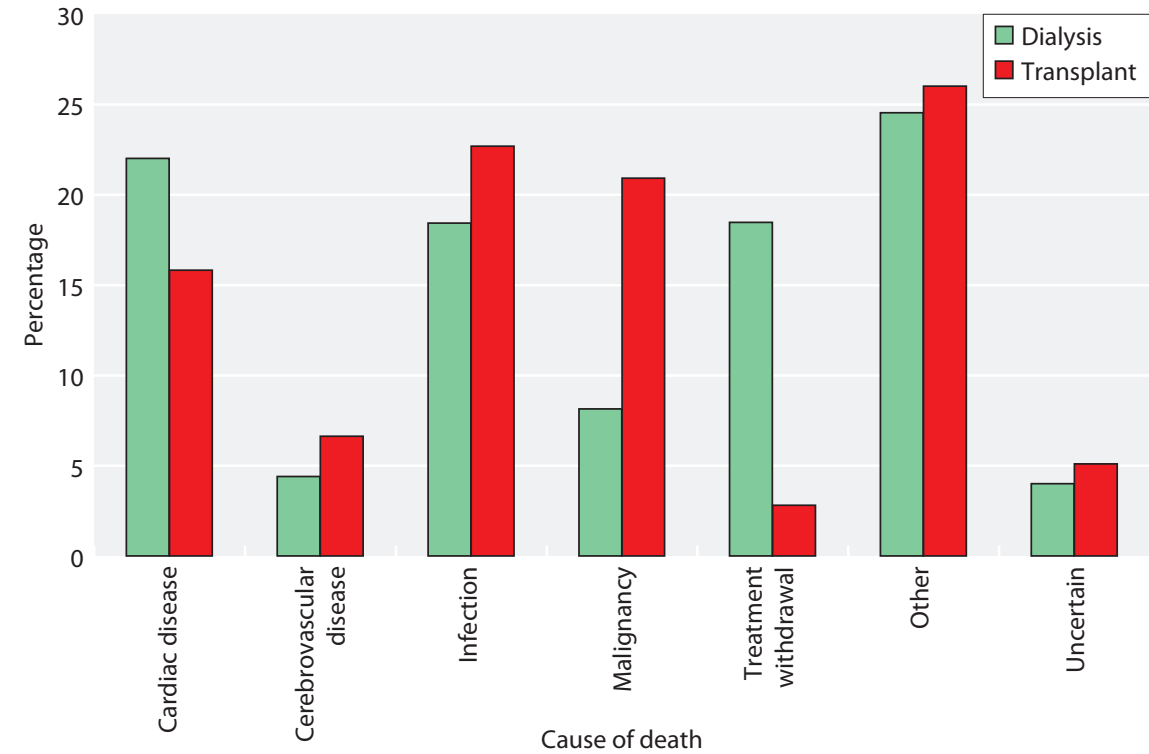

Fig. 3.11. Cause of death by modality for prevalent patients on $1 / 1 / 2011$ deaths in transplant patients attributed to cardiovascular or stroke disease (43\% in 2003 compared to $23 \%$ in 2011) with an increase in the proportion ascribed to infection or malignancy (30\% in 2003 compared to $44 \%$ in 2011). This change has also been reported in other registries, e.g. ANZDATA (http://www.anzdata. org.au) and may reflect better management of cardiovascular risk (although table 3.12 shows BP management remained suboptimal). Explanations for the rising death rate secondary to malignancy may include the increasing age of transplant recipients and the increased intensity of immunosuppressive regimens leading to complications of over-immunosuppression.

Conflicts of interest: Dr I MacPhee has received research funding and speaker honoraria from Astellas.

\section{References}

1 Ansell D, Tomson CRV: UK Renal Registry 11th Annual Report (December 2008) Chapter 15 The UK Renal Registry, UKRR database, validation and methodology. Nephron Clin Pract 2009;111(Suppl. 1): c277-c285
2 MacPhee I, Webb L, Casula A, Udayaraj U: Uk Renal Registry 14th Annual Report (December 2011): Chapter 3 Demographic and biochemistry profile of kidney transplant recipients in the UK in 2010: National and Centre-Specific Analyses. Nephron Clin Pract. 2012;120(suppl 1):c55-79 
3 Bosma RJ, Doorenbos CRC, Stegeman CA, Homan van der Heide JJ, Navis G: Predictive Performance of Renal Function Equations in Renal Transplant Recipients: An analysis of Patient Factors in Bias. Am J Transplant 2005;5:2183-2203

4 Froissart M, Rossert J, Jacquot C, Paillard M, Houillier P: Predictive Performance of the Modification of Diet in Renal Disease and CockcroftGault Equations for Estimating Renal Function. J Am Soc Nephrol. 2005; $16: 763-773$

5 Hariharan, S, McBride MA, Cherikh WS, Tolleris CB, Bresnahan BA, Johnson CP: Post-transplant renal function in the first year predicts long-term kidney transplant survival Kidney Int 2002;62:1:311-318

6 UK Renal Association Clinical Practice Guidelines Committee: Anaemia of CKD, 5th Edition. 2010 http://www.renal.org/clinical/ GuidelinesSection/AnaemiaInCKD.aspx

7 UK Renal Association Clinical Practice Guidelines Committee: Guideline 3.7: Target haemoglobin. 2007 RA Guidelines - Complications of CKD, 4th Edition. 2007. http://www.renal.org/Clinical/GuidelinesSection/ ComplicationsofCKD.aspx

8 UK Renal Association Clinical Practice Guidelines Committee: Guideline: Post-operative Care of the Kidney Transplant Recipient, 5th Edition.
2011 http://www.renal.org/Clinical/GuidelinesSection/Post-operativeCare-Kidney-Transplant-Recipient.aspx

9 UK Renal Association Clinical Practice Guidelines Committee: Guideline 2.1: Treatment of patients with CKD. 2007 RA Guidelines - CKD, 4th Edition. 2007. http://www.renal.org/Clinical/GuidelinesSection/CKD.aspx 10 White CA, Akbari A, Doucette S, Fergusson D, Knoll GA: Estimating Glomerular Filtration Rate in Kidney Transplantation: Is the New Chronic Kidney Disease Epidemiology Collaboration Equation Any Better? Clin Chem 2010;56:3:474-477

11 Ng FL, Holt DW, Chang RWS, MacPhee IAM: Black renal transplant recipients have poorer long-term graft survival than CYP3A5 expressers from other ethnic groups. Nephrol Dial Transplant 2010;25:628-634

12 Isaacs RB, Nock SL, Spencer CE, Connors AF Jr, Wang XQ, Sawyer R, Lobo PI: Racial disparities in renal transplant outcomes. Am J Kidney Dis 1999;34:4:706-712

13 Udayaraj U, Casula A, Ansell D, Dudley CRK, Ravanan R: Chronic Kidney Disease in Transplant Recipients - Is It Different From Chronic Native Kidney Disease? Transplantation 2010;90:7:765-770 